\title{
Absolute Properties of the Eclipsing Triple Star Co Andromedae: Constraints on Convective Core Overshooting
}

\section{Citation}

Lacy, Claud H. Sandberg, Guillermo Torres, Antonio Claret, David Charbonneau, Francis T. O'Donovan, and Georgi Mandushev. 2010. "ABSOLUTE PROPERTIES OF THE ECLIPSING TRIPLE STAR CO ANDROMEDAE: CONSTRAINTS ON CONVECTIVE CORE OVERSHOOTING." The Astronomical Journal 139 (6): 2347-59. https://doi.org/10.1088/0004-6256/139/6/2347.

\section{Permanent link}

http://nrs.harvard.edu/urn-3:HUL.InstRepos:41412235

\section{Terms of Use}

This article was downloaded from Harvard University's DASH repository, and is made available under the terms and conditions applicable to Other Posted Material, as set forth at http:// nrs.harvard.edu/urn-3:HUL.InstRepos:dash.current.terms-of-use\#LAA

\section{Share Your Story}

The Harvard community has made this article openly available.

Please share how this access benefits you. Submit a story.

\section{Accessibility}




\title{
ABSOLUTE PROPERTIES OF THE ECLIPSING TRIPLE STAR CO ANDROMEDAE: CONSTRAINTS ON CONVECTIVE CORE OVERSHOOTING
}

\author{
Claud H. Sandberg Lacy ${ }^{1}$, Guillermo Torres ${ }^{2}$, Antonio Claret ${ }^{3}$, David Charbonneau ${ }^{2}$, Francis T. O’Donovan ${ }^{4}$, \\ AND GEORGi MANDUSHEV ${ }^{5}$ \\ ${ }^{1}$ Physics Department, University of Arkansas, Fayetteville, AR 72701, USA; clacy@uark.edu \\ ${ }^{2}$ Harvard-Smithsonian Center for Astrophysics, 60 Garden Street, Cambridge, MA 02138, USA; gtorres@cfa.harvard.edu, dcharbonneau@cfa.harvard.edu \\ ${ }^{3}$ Instituto de Astrofisica de Andalucia, CSIC, Apdo. 3004, E-18080 Granada, Spain; claret@iaa.es \\ ${ }^{4}$ NASA Postdoctoral Program Fellow, Goddard Space Flight Center, 8800 Greenbelt Road, Greenbelt, MD 20771, USA; ftod@caltech.edu \\ ${ }^{5}$ Lowell Observatory, 1400 West Mars Hill Road, Flagstaff, AZ 86001, USA; gmand@ lowell.edu \\ Received 2010 March 1; accepted 2010 March 25; published 2010 April 26
}

\begin{abstract}
Accurate absolute properties have been determined for the eclipsing triple star $\mathrm{CO}$ And (F8+F8) based on extensive differential photometry obtained by three robotic observatories and CfA spectroscopy. The eclipsing binary star orbit is circular with a period of 3.655 days. The triple nature of this system is revealed by more than a century of timings of minimum light, and by the presence of third light in the photometric orbits. The masses of the eclipsing pair are $1.289 \pm 0.007$ and $1.264 \pm 0.007$ solar masses, and the corresponding radii are $1.727 \pm 0.021$ and $1.694 \pm 0.017$ solar radii. These stars are synchronously rotating and are near the end of their main-sequence phase, at an age of about 3.6 Gyr. The much fainter widely separated third body appears to have a mass of about 0.8 solar masses. The distance to the system is $377 \pm 25 \mathrm{pc}$.

Key words: binaries: eclipsing - binaries: spectroscopic - stars: fundamental parameters - stars: individual (CO And) - stars: rotation - stars: solar-type
\end{abstract}

Online-only material: machine-readable and VO tables

\section{INTRODUCTION}

The photometric variability of $\mathrm{CO}$ And $\left(\mathrm{BD}+46^{\circ} 281\right.$, TYC $3268-398-1 ; \alpha=01^{\mathrm{h}} 11^{\mathrm{m}} 24.83, \delta=+46^{\circ} 57^{\prime} 49^{\prime} \cdot 3, \mathrm{~J} 2000.0$; Sp F8+F8 V, $V=10.70-11.22$ ) was announced by Geyer et al. (1955) from photographic measures, with the notation that it might be an eclipsing variable star. Shortly thereafter, Grigorewski (1956) measured times of four minima photographically and gave an eclipse ephemeris with a period of 11.0436 days. The Bamberg observers published their own times of eclipse (Reim 1957) and found a period of 1.827633 days. Dates of minima predicted on this basis were then measured photographically, visually, and with CCD detectors. Lacy guessed in 2002 December that the true period was probably twice this value since there was no sign of a secondary eclipse in his accurate differential photometry obtained with the URSA WebScope (Lacy 2003a), and later CfA spectroscopic work (below) has confirmed that the true orbital period is almost exactly twice the Reim (1957) value. In 2003, Charbonneau and collaborators began monitoring selected star fields looking for transits of exoplanets similar to those of HD 209458b. One of the fields that was measured serendipitously contained $\mathrm{CO}$ And. The positional coincidence was discovered in 2004 December, and we were able to extract very accurate photometry of the eclipsing binary star measured by the Sleuth telescope, part of the Trans-Atlantic Exoplanet Survey (O'Donovan et al. 2004). The combination of photometric and spectroscopic analyses of our accurate data enables the determination of precise absolute properties of the component stars and provides strong constraints on theories of stellar evolution.

\section{OBSERVATIONS AND REDUCTIONS}

\subsection{Differential Photometric Observations}

Our photometric work on CO And began in 2001 and is based on observations obtained with three robotic instruments: the
URSA WebScope at Kimpel Observatory (Lacy et al. 2001) on the campus of the University of Arkansas, the NFO WebScope at the NF/ Observatory (NFO; Grauer et al. 2008) near Silver City, NM, and the Sleuth telescope (O'Donovan et al. 2004) in California.

Kimpel Observatory (http://ursa.uark.edu) consists of a Meade 10 inch f/6.3 LX-200 telescope with a Santa Barbara Instruments Group ST8 CCD camera (binned $2 \times 2$ to produce $765 \times 510$ pixel images with $2.3 \operatorname{arcsec}^{2}$ pixels) inside a Technical Innovations Robo-Dome, and controlled automatically by an Apple Macintosh G4 computer. The observatory is located on top of Kimpel Hall on the Fayetteville campus of the University of Arkansas, with the control room directly beneath the observatory inside the building. Exposures of 60 or $120 \mathrm{~s}$ made through a Bessell $V$ filter (2.0 mm of GG 495 and $3.0 \mathrm{~mm}$ of BG 39) were read out and downloaded by ImageGrabber (camera control software written by J. Sabby) to the control computer over a $30 \mathrm{~s}$ interval, then the next exposure was begun. The observing cadence was therefore about 90-150 s per observation. The variable star would sometimes be monitored continuously for 4-6 hr. CO And was observed by URSA on 104 nights during parts of nine observing seasons from 2001 December 2 to 2009 February 22. The total number of URSA observations is 5941. The frames were analyzed by a virtual measuring engine application written by Lacy that flat-fielded the images, automatically located the variable and comparison stars in the image, measured their brightnesses, subtracted the corresponding sky brightness, and corrected for the differences in airmass between the stars. Extinction coefficients were determined nightly from the comparison star measurements. They averaged $0.25 \mathrm{mag}^{\text {airmass }}{ }^{-1}$. CO And is TYC 3268-0398-1. The comparison stars were TYC 3268-0400-1 (comp; $\left.V_{T}=10.31, B_{T}-V_{T}=+1.24\right)$ as listed in the Tycho-2 Catalog; = Høg et al. 2000), and TYC 3268-0958-1 (ck; $V_{T}=11.38, B_{T}-V_{T}=+1.44$ ). In measuring the URSA images, a square aperture with 30 arcsec sides was used. Both comparison stars are within 10 arcmin of the variable star (var). 

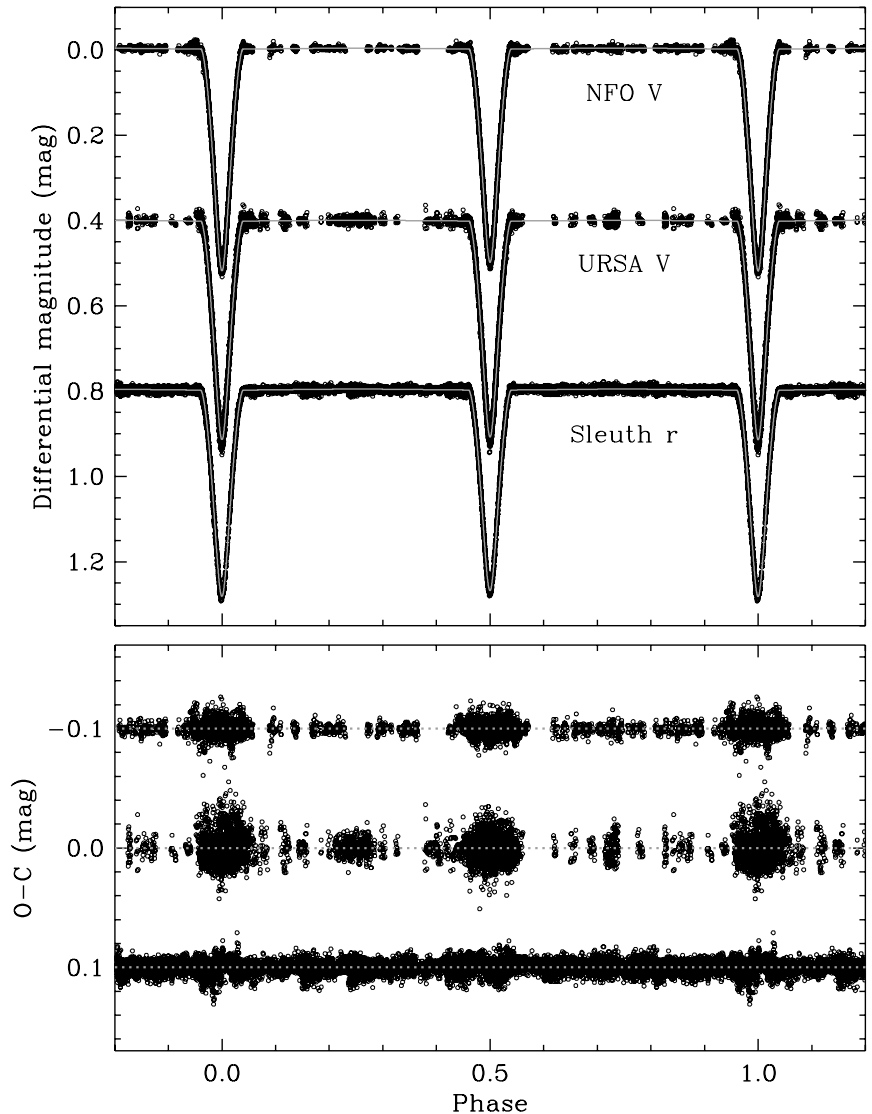

Figure 1. Differential photometry for CO And obtained with the NFO, URSA, and Sleuth telescopes, along with our best-fit models. Residuals are shown at the bottom, with NFO and Sleuth displaced for clarity.

Table 1

Differential Photometric Observations of CO And in $V$ Made with the URSA WebScope

\begin{tabular}{ccc}
\hline \hline Phase & $V(\mathrm{mag})$ & HJD-2,400,000 \\
\hline 0.48295 & 0.731 & 52245.58856 \\
0.48320 & 0.739 & 52245.58948 \\
0.48345 & 0.733 & 52245.59039 \\
0.48370 & 0.739 & 52245.59132 \\
0.48395 & 0.752 & 52245.59224 \\
\hline
\end{tabular}

(This table is available in its entirety in machine-readable and Virtual Observatory (VO) forms in the online journal. A portion is shown here for guidance regarding its form and content.)

The comparison star magnitude differences (comp-ck) were constant at the level of 0.004 mag (standard deviation within a night), and $0.013 \mathrm{mag}$ for the standard deviation of the nightly mean magnitude difference. The URSA differential magnitude (var-comp) of the variable star was referenced only to the magnitude of the comparison star, comp. The resulting 5941 URSA $V$ magnitude differences (var-comp) are listed in Table 1 (without any nightly corrections) and plotted in Figures 1-3 (after the nightly corrections discussed below have been applied). The eclipse depths are 0.476 mag for the primary and 0.475 mag for the secondary. The precision of the raw variable star differential magnitudes is about $0.012 \mathrm{mag}$.

Another telescope we used is the NFO WebScope, a refurbished 24 inch Group 128 Cassegrain reflector with a $2 \mathrm{k} \times 2 \mathrm{k}$ Kodak CCD camera, located near Silver City, NM. Observations consisted of $120 \mathrm{~s}$ exposures through a Bessell $V$ filter. $\mathrm{CO}$
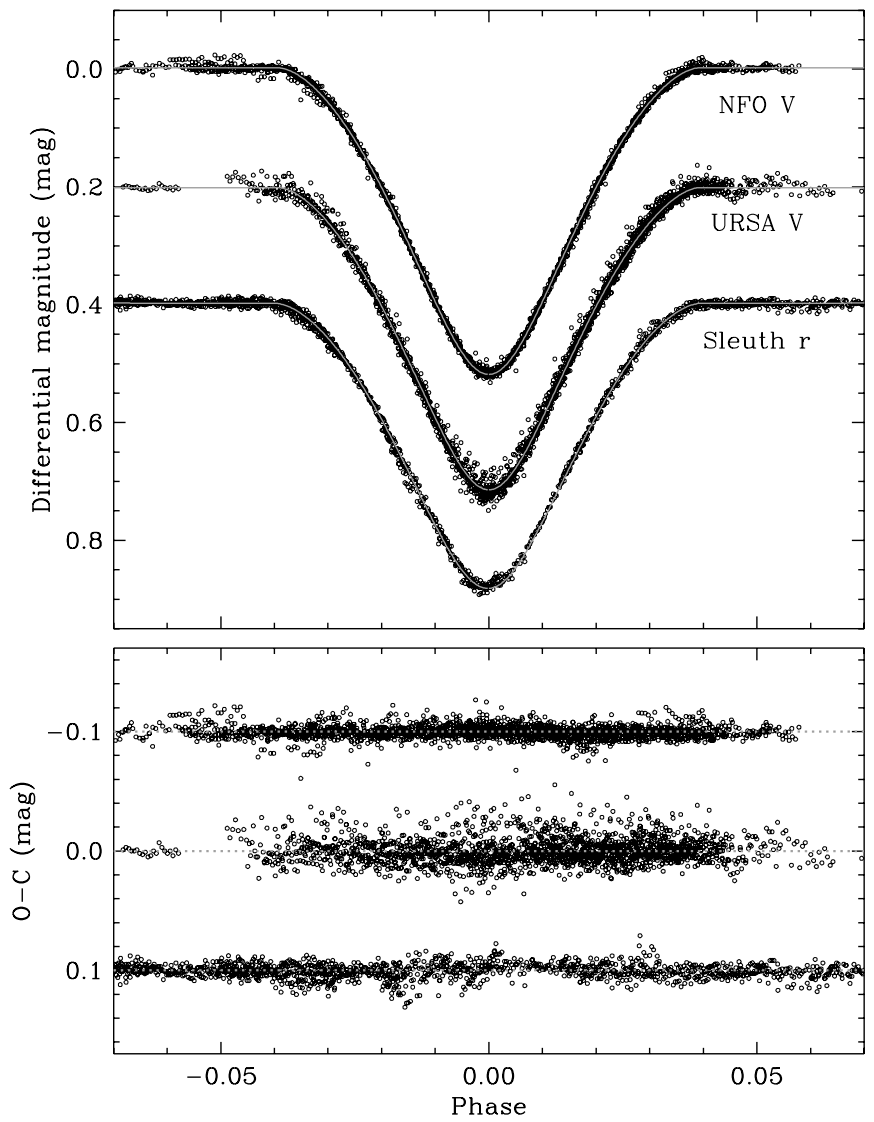

Figure 2. Same as Figure 1, showing an enlarged view of the primary (deeper) eclipse.
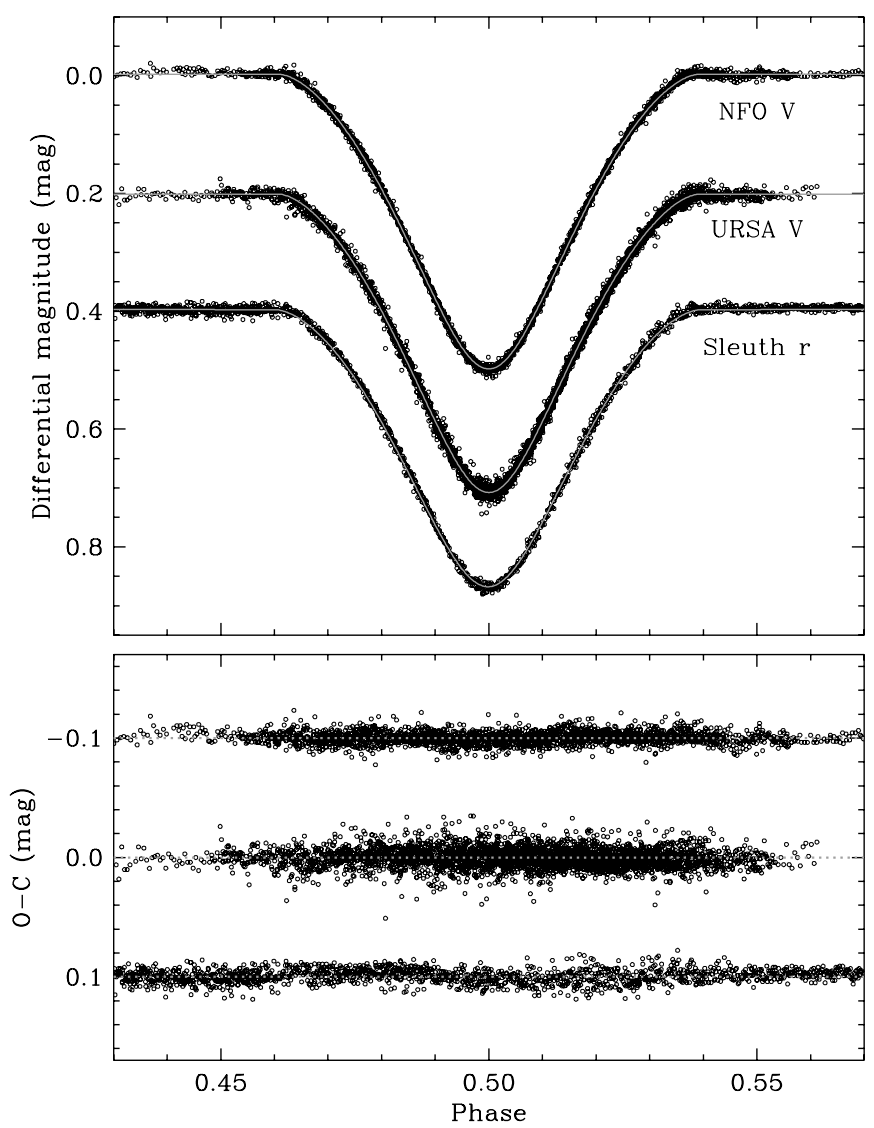

Figure 3. Same as Figure 1, showing an enlarged view of the secondary (shallower) eclipse. 
Table 2

Differential Photometric Observations of CO And in $V$ Made with the NFO WebScope

\begin{tabular}{ccr}
\hline \hline Phase & $V(\mathrm{mag})$ & HJD-2,400,000 \\
\hline 0.51444 & 1.100 & 53568.93531 \\
0.51501 & 1.093 & 53568.93740 \\
0.51558 & 1.083 & 53568.93949 \\
0.51616 & 1.067 & 53568.94159 \\
0.51673 & 1.057 & 53568.94368 \\
\hline
\end{tabular}

(This table is available in its entirety in machine-readable and Virtual Observatory (VO) forms in the online journal. A portion is shown here for guidance regarding its form and content.)

And was observed by NFO on 143 nights during parts of five observing seasons from 2005 July 17 to 2009 February 15 , yielding 5025 observations. Extinction coefficients were determined nightly from the comparison star measurements. They averaged $0.18 \mathrm{mag}$ airmass $^{-1}$. The same comparison stars as those of the URSA telescope were used. In measuring the NFO images, a square aperture with 23 arcsec sides was used. The comparison star magnitude differences (comp-ck) were constant at the level of $0.007 \mathrm{mag}$ (standard deviation within a night), and $0.010 \mathrm{mag}$ for the standard deviation of the nightly mean magnitude difference. The differential magnitude (var-comps) of the variable star was referenced to the magnitude corresponding to the sum of the fluxes of the comparison star, comp, and the check star, ck. The resulting $5025 \mathrm{~V}$ magnitude differences (var-comps) are listed in Table 2 (without nightly corrections) and plotted in Figures 1-3 (including nightly corrections; see below). The precision of the variable star differential magnitudes is about 0.010 mag.

We noticed early on during the observations that the NFO magnitudes showed a small but significant offset from night to night, on the order of a hundredth of a magnitude. The origin of the offset is a variation in responsivity across the field of view of the NFO combined with imprecise centering from night to night. These variations are a well-known effect of the optics when using wide-field imaging telescopes such as the NFO. We have removed most of this variation by using dithered exposures of open clusters to measure this variation, fitting a two-dimensional polynomial (see Selman 2004), and subtracting the variation during initial reductions (photometric flat). The URSA observations, on the other hand, show this kind of effect to a much smaller extent. We have removed these nightly offsets before further analysis by using a procedure discussed in Section 3. The Sleuth photometry in Table 3 (9289 Sloan $r$ magnitudes) was found to be free of these effects, though the raw data contained a number of clearly aberrant observations, which have been removed. A circular aperture of radius 30 arcsec was used in measuring differential magnitudes with Sleuth (O’Donovan et al. 2004).

\subsection{Ephemeris}

From the differential photometry of the three different telescopes, we have determined very accurate times of eclipse by using the method of Kwee \& van Woerden (1956). We list these and other times of eclipse from the literature in Table 4 . The minima now total 188. Uncertainties were taken from the literature, or were estimated for the older methods. We have adopted the usual convention that the deeper eclipse is the primary eclipse (type 1) and the other eclipse is the secondary eclipse (type 2). In the case of CO And, both eclipses are nearly the same depth,

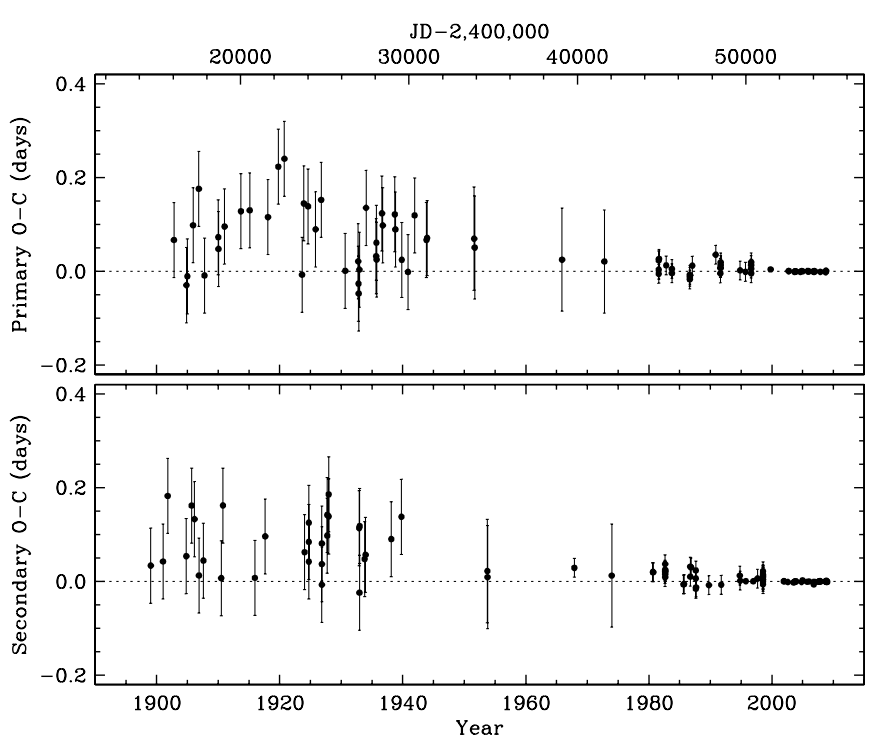

Figure 4. Residuals from a linear eclipse ephemeris derived from recent very accurate CCD eclipse timings (years 2003-2009). The top panel shows results from the dates of primary eclipses, and the bottom panel from the dates of secondary eclipses. In both cases, the residuals are significantly positive prior to 1940 , during the era of photographic eclipse timings. We believe that these positive residuals are a light travel time effect across an orbit with a third component in the system.

Table 3

Differential Photometric Observations of CO And in Sloan $r$ Made with the Sleuth Telescope

\begin{tabular}{ccc}
\hline \hline Phase & $r(\mathrm{mag})$ & HJD-2,400,000 \\
\hline 0.00006 & 0.479 & 52890.81791 \\
0.00014 & 0.469 & 52934.68226 \\
0.00020 & 0.473 & 52901.78444 \\
0.00030 & 0.481 & 52923.71684 \\
0.00042 & 0.479 & 52890.81925 \\
\hline
\end{tabular}

(This table is available in its entirety in machine-readable and Virtual Observatory (VO) forms in the online journal. A portion is shown here for guidance regarding its form and content.)

but our differential photometry can distinguish them. The primary eclipse corresponds to the eclipse by the cooler star, which we refer to as A or c, of the hotter star, which we refer to as B or h. It turns out that $\mathrm{A}=\mathrm{c}$, the cooler star, is the more massive star (see below). We avoid referring to the stars as the primary or secondary component since this term is ambiguous in this system.

When we fitted a linear ephemeris to the dates of minimum light, we found that the modern, much more accurate CCD dates gave a different ephemeris than the older minima. We therefore used only the more recent (2003-2009) minima to determine an eclipse ephemeris to use in further analysis of our recently measured data:

$$
\text { HJD Min I = 2, 453, 764.44301(17) + 3.65533601(91), }
$$

where the uncertainties in the last digits are given in parentheses. The eclipse timing residuals based on this ephemeris are shown in Figure 4. The significantly positive mean residuals prior to year 1940 are likely due to the light travel time across an orbit with a third body. Thus, one should expect that third light might be present in the photometric analysis, which it is. 
Table 4

Dates of Minimum Light for CO And

\begin{tabular}{|c|c|c|c|c|c|}
\hline $\begin{array}{c}\text { HJD } \\
-2,400,000 \\
\end{array}$ & $\begin{array}{c}\text { Eclipse } \\
\text { Type }\end{array}$ & $\begin{array}{c}\text { Uncertainty } \\
\text { (days) }\end{array}$ & $\begin{array}{c}O-C \\
\text { (days) }\end{array}$ & Method & Reference \\
\hline 14672.48600 & 2 & 0.08000 & 0.03395 & $\mathrm{pg}$ & 1 \\
\hline 15403.56200 & 2 & 0.08000 & 0.04275 & pg & 1 \\
\hline 15677.85200 & 2 & 0.08000 & 0.18255 & pg & 1 \\
\hline 16048.75300 & 1 & 0.08000 & 0.06694 & $\mathrm{pg}$ & 1 \\
\hline 16781.63500 & 2 & 0.08000 & 0.05407 & pg & 1 \\
\hline 16790.69000 & 1 & 0.08000 & 0.02927 & pg & 1 \\
\hline 16845.53900 & 1 & 0.08000 & 0.01031 & pg & 1 \\
\hline 17099.75700 & 2 & 0.08000 & 0.16184 & pg & 1 \\
\hline 17185.59400 & 1 & 0.08000 & 0.09844 & pg & 1 \\
\hline 17271.52900 & 2 & 0.08000 & 0.13305 & pg & 1 \\
\hline 17525.61800 & 1 & 0.08000 & 0.17619 & pg & 1 \\
\hline 17534.59300 & 2 & 0.08000 & 0.01285 & pg & 1 \\
\hline 17797.80900 & 2 & 0.08000 & 0.04466 & pg & 1 \\
\hline 17861.72400 & 1 & 0.08000 & 0.00872 & pg & 1 \\
\hline 18680.60100 & 1 & 0.08000 & 0.07301 & pg & 1 \\
\hline 18691.54200 & 1 & 0.08000 & 0.04801 & pg & 1 \\
\hline 18857.81900 & 2 & 0.08000 & 0.00722 & pg & 1 \\
\hline 18956.66800 & 2 & 0.08000 & 0.16215 & pg & 1 \\
\hline 19053.46800 & 1 & 0.08000 & 0.09574 & pg & 1 \\
\hline 20025.82000 & 1 & 0.08000 & 0.12836 & $\mathrm{pg}$ & 1 \\
\hline 20548.53500 & 1 & 0.08000 & 0.13031 & $\mathrm{pg}$ & 1 \\
\hline 20853.63300 & 2 & 0.08000 & 0.00776 & $\mathrm{pg}$ & 1 \\
\hline 21467.81800 & 2 & 0.08000 & 0.09631 & pg & 1 \\
\hline 21630.50000 & 1 & 0.08000 & 0.11585 & pg & 1 \\
\hline 22244.70400 & 1 & 0.08000 & 0.22340 & $\mathrm{pg}$ & 1 \\
\hline 22606.59900 & 1 & 0.08000 & 0.24014 & pg & 1 \\
\hline 23651.77800 & 1 & 0.08000 & 0.00696 & $\mathrm{pg}$ & 1 \\
\hline 23761.59000 & 1 & 0.08000 & 0.14496 & $\mathrm{pg}$ & 1 \\
\hline 23803.54400 & 2 & 0.08000 & 0.06260 & pg & 1 \\
\hline 24013.80200 & 1 & 0.08000 & 0.13878 & pg & 1 \\
\hline 24055.74200 & 2 & 0.08000 & 0.04241 & pg & 1 \\
\hline 24055.78400 & 2 & 0.08000 & 0.08441 & pg & 1 \\
\hline 24055.82500 & 2 & 0.08000 & 0.12541 & pg & 1 \\
\hline 24459.70400 & 1 & 0.08000 & 0.08978 & pg & 1 \\
\hline 24788.74700 & 1 & 0.08000 & 0.15254 & pg & 1 \\
\hline 24830.62400 & 2 & 0.08000 & 0.00682 & pg & 1 \\
\hline 24830.66800 & 2 & 0.08000 & 0.03718 & pg & 1 \\
\hline 24830.71200 & 2 & 0.08000 & 0.08118 & pg & 1 \\
\hline 25148.74300 & 2 & 0.08000 & 0.09794 & pg & 1 \\
\hline 25148.78700 & 2 & 0.08000 & 0.14194 & pg & 1 \\
\hline 25236.51200 & 2 & 0.08000 & 0.13888 & pg & 1 \\
\hline 25236.55900 & 2 & 0.08000 & 0.18588 & pg & 1 \\
\hline 26217.83200 & 1 & 0.08000 & 0.00116 & pg & 1 \\
\hline 26985.47300 & 1 & 0.08000 & 0.02160 & pg & 2 \\
\hline 27003.70200 & 1 & 0.08000 & 0.02608 & pg & 1 \\
\hline 27014.64700 & 1 & 0.08000 & 0.04709 & pg & 1 \\
\hline 27042.22300 & 2 & 0.08000 & 0.11389 & $\mathrm{pg}$ & 2 \\
\hline 27058.56200 & 1 & 0.08000 & 0.00388 & pg & 1 \\
\hline 27060.36200 & 2 & 0.08000 & 0.02379 & pg & 2 \\
\hline 27071.47000 & 2 & 0.08000 & 0.11820 & $\mathrm{pg}$ & 1 \\
\hline 27367.48200 & 2 & 0.08000 & 0.04799 & $\mathrm{pg}$ & 2 \\
\hline 27422.32100 & 2 & 0.08000 & 0.05695 & $\mathrm{pg}$ & 2 \\
\hline 27453.47000 & 1 & 0.08000 & 0.13559 & $\mathrm{pg}$ & 1 \\
\hline 28052.84200 & 1 & 0.08000 & 0.03248 & pg & 1 \\
\hline 28067.49200 & 1 & 0.08000 & 0.06114 & $\mathrm{pg}$ & 2 \\
\hline 28078.42200 & 1 & 0.08000 & 0.02513 & $\mathrm{pg}$ & 2 \\
\hline 28396.53500 & 1 & 0.08000 & 0.12390 & $\mathrm{pg}$ & 2 \\
\hline 28447.68400 & 1 & 0.08000 & 0.09820 & pg & 1 \\
\hline 28950.28500 & 2 & 0.08000 & 0.09049 & pg & 2 \\
\hline 29160.49800 & 1 & 0.08000 & 0.12167 & pg & 2 \\
\hline 29193.36400 & 1 & 0.08000 & 0.08965 & pg & 2 \\
\hline 29553.46300 & 2 & 0.08000 & 0.13805 & pg & 2 \\
\hline 29573.45400 & 1 & 0.08000 & 0.02470 & pg & 2 \\
\hline 29942.61700 & 1 & 0.08000 & 0.00123 & pg & 1 \\
\hline 30337.51400 & 1 & 0.08000 & 0.11948 & pg & 1 \\
\hline 31035.63100 & 1 & 0.08000 & 0.06730 & pg & 1 \\
\hline 31079.49900 & 1 & 0.08000 & 0.07127 & pg & 1 \\
\hline
\end{tabular}

Table 4

(Continued)

\begin{tabular}{|c|c|c|c|c|c|}
\hline $\begin{array}{c}\text { HJD } \\
-2,400,000 \\
\end{array}$ & $\begin{array}{l}\text { Eclipse } \\
\text { Type }\end{array}$ & $\begin{array}{c}\text { Uncertainty } \\
\text { (days) }\end{array}$ & $\begin{array}{c}O-C \\
\text { (days) }\end{array}$ & Method & Reference \\
\hline 33868.51900 & 1 & 0.11000 & 0.06989 & pg & 3 \\
\hline 33901.39800 & 1 & 0.11000 & 0.05087 & $\mathrm{pg}$ & 3 \\
\hline 34652.54100 & 2 & 0.11000 & 0.02232 & pg & 3 \\
\hline 34663.49400 & 2 & 0.11000 & 0.00931 & pg & 3 \\
\hline 39088.29400 & 1 & 0.11000 & 0.02507 & pg & 4 \\
\hline 39821.19300 & 2 & 0.02000 & 0.02920 & vis & 5 \\
\hline 41599.50600 & 1 & 0.11000 & 0.02123 & pg & 4 \\
\hline 42036.31000 & 2 & 0.11000 & 0.01258 & $\mathrm{pg}$ & 4 \\
\hline 44485.39200 & 2 & 0.02000 & 0.01945 & vis & 6 \\
\hline 44485.39300 & 2 & 0.02000 & 0.02045 & vis & 6 \\
\hline 44823.48610 & 1 & 0.02000 & 0.00503 & vis & 7 \\
\hline 44823.49500 & 1 & 0.02000 & 0.00387 & vis & 7 \\
\hline 44823.51500 & 1 & 0.02000 & 0.02387 & vis & 7 \\
\hline 44845.45000 & 1 & 0.02000 & 0.02685 & vis & 7 \\
\hline 45205.48300 & 2 & 0.02000 & 0.00926 & vis & 7 \\
\hline 45205.48950 & 2 & 0.02000 & 0.01576 & vis & 7 \\
\hline 45205.49440 & 2 & 0.02000 & 0.02066 & vis & 7 \\
\hline 45205.49640 & 2 & 0.02000 & 0.02266 & vis & 7 \\
\hline 45205.49900 & 2 & 0.02000 & 0.02526 & vis & 7 \\
\hline 45205.51100 & 2 & 0.02000 & 0.03726 & vis & 7 \\
\hline 45269.45500 & 1 & 0.02000 & 0.01288 & vis & 8 \\
\hline 45609.38470 & 1 & 0.02000 & 0.00367 & vis & 7 \\
\hline 45609.39370 & 1 & 0.02000 & 0.00533 & vis & 7 \\
\hline 46298.41300 & 2 & 0.02000 & 0.00621 & vis & 9 \\
\hline 46331.31200 & 2 & 0.02000 & 0.00523 & vis & 9 \\
\hline 46669.41890 & 1 & 0.02000 & 0.01691 & vis & 10 \\
\hline 46669.42440 & 1 & 0.02000 & 0.01141 & vis & 10 \\
\hline 46669.42900 & 1 & 0.02000 & 0.00681 & vis & 10 \\
\hline 46700.51650 & 2 & 0.02000 & 0.01033 & vis & 11 \\
\hline 46700.53800 & 2 & 0.02000 & 0.03183 & vis & 11 \\
\hline 46744.40030 & 2 & 0.02000 & 0.03010 & vis & 11 \\
\hline 46819.31700 & 1 & 0.02000 & 0.01241 & vis & 12 \\
\hline 47029.47100 & 2 & 0.02000 & 0.01541 & vis & 13 \\
\hline 47029.49300 & 2 & 0.02000 & 0.00659 & vis & 13 \\
\hline 47029.51000 & 2 & 0.02000 & 0.02359 & vis & 13 \\
\hline 47040.44000 & 2 & 0.02000 & 0.01242 & vis & 13 \\
\hline 47804.41000 & 2 & 0.02000 & 0.00765 & vis & 14 \\
\hline 48208.36800 & 1 & 0.02000 & 0.03573 & vis & 15 \\
\hline 48482.47830 & 1 & 0.02000 & 0.00418 & vis & 16 \\
\hline 48482.49000 & 1 & 0.02000 & 0.00752 & vis & 16 \\
\hline 48504.42200 & 1 & 0.02000 & 0.00751 & vis & 16 \\
\hline 48504.42800 & 1 & 0.02000 & 0.01351 & vis & 17 \\
\hline 48504.43400 & 1 & 0.02000 & 0.01951 & vis & 16 \\
\hline 48535.47800 & 2 & 0.02000 & 0.00685 & vis & 16 \\
\hline 49639.40900 & 2 & 0.02000 & 0.01268 & vis & 18 \\
\hline 49659.50300 & 1 & 0.02000 & 0.00233 & vis & 18 \\
\hline 49661.33000 & 2 & 0.02000 & 0.00166 & vis & 18 \\
\hline 49977.51400 & 1 & 0.02000 & 0.00090 & vis & 18 \\
\hline 49997.62000 & 2 & 0.00100 & 0.00075 & $\operatorname{ccd}$ & 19 \\
\hline 50317.45720 & 1 & 0.02000 & 0.00395 & vis & 20 \\
\hline 50317.46700 & 1 & 0.02000 & 0.00585 & vis & 20 \\
\hline 50317.47460 & 1 & 0.02000 & 0.01345 & vis & 20 \\
\hline 50317.48080 & 1 & 0.02000 & 0.01965 & vis & 20 \\
\hline 50436.26010 & 2 & 0.00100 & 0.00053 & $\operatorname{ccd}$ & 21 \\
\hline 50688.48400 & 2 & 0.02000 & 0.00624 & vis & 20 \\
\hline 51017.45220 & 2 & 0.02000 & 0.00580 & vis & 20 \\
\hline 51017.45770 & 2 & 0.02000 & 0.00030 & vis & 20 \\
\hline 51017.46190 & 2 & 0.02000 & 0.00390 & vis & 20 \\
\hline 51017.46260 & 2 & 0.02000 & 0.00460 & vis & 20 \\
\hline 51017.46750 & 2 & 0.02000 & 0.00950 & vis & 20 \\
\hline 51017.46890 & 2 & 0.02000 & 0.01090 & vis & 20 \\
\hline 51017.47160 & 2 & 0.02000 & 0.01360 & vis & 20 \\
\hline 51017.47300 & 2 & 0.02000 & 0.01500 & vis & 20 \\
\hline 51017.48000 & 2 & 0.02000 & 0.02200 & vis & 20 \\
\hline 51465.24100 & 1 & 0.00100 & 0.00434 & $\mathrm{ccd}$ & 22 \\
\hline 52245.65158 & 2 & 0.00016 & 0.00068 & URSA & 23 \\
\hline 52267.58334 & 2 & 0.00011 & 0.00043 & URSA & 23 \\
\hline
\end{tabular}


Table 4

(Continued)

\begin{tabular}{|c|c|c|c|c|c|}
\hline $\begin{array}{c}\text { HJD } \\
-2,400,000 \\
\end{array}$ & $\begin{array}{c}\text { Eclipse } \\
\text { Type }\end{array}$ & $\begin{array}{c}\text { Uncertainty } \\
\text { (days) }\end{array}$ & $\begin{array}{c}O-C \\
\text { (days) }\end{array}$ & Method & Reference \\
\hline 52490.55720 & 2 & 0.00020 & 0.00121 & $\operatorname{ccd}$ & 24 \\
\hline 52539.90630 & 1 & 0.00030 & 0.00085 & $\operatorname{ccd}$ & 25 \\
\hline 52826.84740 & 2 & 0.00050 & 0.00192 & URSA & 26 \\
\hline 52888.98908 & 2 & 0.00017 & 0.00096 & Sle & 27 \\
\hline 52890.81667 & 1 & 0.00010 & 0.00103 & Sle & 27 \\
\hline 52899.95568 & 2 & 0.00016 & 0.00036 & Sle & 27 \\
\hline 52901.78355 & 1 & 0.00011 & 0.00016 & Sle & 27 \\
\hline 52910.92212 & 2 & 0.00012 & 0.00007 & Sle & 27 \\
\hline 52934.68220 & 1 & 0.00018 & 0.00046 & URSA & 26 \\
\hline 52965.75230 & 2 & 0.00030 & 0.00021 & URSA & 26 \\
\hline 52976.71844 & 2 & 0.00013 & 0.00034 & Sle & 27 \\
\hline 52982.20090 & 1 & 0.00010 & 0.00020 & $\operatorname{ccd}$ & 28 \\
\hline 53230.76320 & 1 & 0.00030 & 0.00075 & URSA & 29 \\
\hline 53302.04200 & 2 & 0.00020 & 0.00100 & $\operatorname{ccd}$ & 30 \\
\hline 53314.83760 & 1 & 0.00030 & 0.00092 & URSA & 31 \\
\hline 53358.70090 & 1 & 0.00050 & 0.00019 & URSA & 31 \\
\hline 53360.53090 & 2 & 0.00040 & 0.00252 & URSA & 31 \\
\hline 53634.67810 & 2 & 0.00030 & 0.00048 & URSA & 31 \\
\hline 53676.71610 & 1 & 0.00040 & 0.00115 & URSA & 31 \\
\hline 53687.68078 & 1 & 0.00020 & 0.00017 & NFO & 31 \\
\hline 53731.54510 & 1 & 0.00020 & 0.00011 & URSA & 32 \\
\hline 53751.64940 & 2 & 0.00030 & 0.00007 & URSA & 32 \\
\hline 54016.66120 & 1 & 0.00040 & 0.00001 & URSA & 32 \\
\hline 54027.62700 & 1 & 0.00030 & 0.00020 & NFO & 32 \\
\hline 54029.44880 & 2 & 0.00100 & 0.00607 & $\operatorname{ccd}$ & 33 \\
\hline 54038.59240 & 1 & 0.00050 & 0.00081 & URSA & 32 \\
\hline 54045.90390 & 1 & 0.00030 & 0.00002 & NFO & 32 \\
\hline 54047.73100 & 2 & 0.00020 & 0.00055 & NFO & 32 \\
\hline 54067.83650 & 1 & 0.00050 & 0.00060 & NFO & 32 \\
\hline 54080.62810 & 2 & 0.00020 & 0.00147 & $\mathrm{NFO}$ & 32 \\
\hline 54100.73420 & 1 & 0.00020 & 0.00028 & NFO & 32 \\
\hline 54321.88200 & 2 & 0.00030 & 0.00025 & URSA & 34 \\
\hline 54332.84740 & 2 & 0.00030 & 0.00036 & NFO & 34 \\
\hline 54343.81310 & 2 & 0.00030 & 0.00067 & URSA & 34 \\
\hline 54365.74560 & 2 & 0.00030 & 0.00018 & URSA & 34 \\
\hline 54365.74560 & 2 & 0.00020 & 0.00018 & NFO & 34 \\
\hline 54387.67850 & 2 & 0.00020 & 0.00070 & $\mathrm{NFO}$ & 34 \\
\hline 54409.61030 & 2 & 0.00030 & 0.00048 & URSA & 34 \\
\hline 54418.74740 & 1 & 0.00040 & 0.00076 & NFO & 34 \\
\hline 54420.57600 & 2 & 0.00050 & 0.00018 & URSA & 34 \\
\hline 54734.93520 & 2 & 0.00030 & 0.00048 & NFO & 34 \\
\hline 54736.76080 & 1 & 0.00030 & 0.00159 & URSA & 34 \\
\hline 54745.90020 & 2 & 0.00030 & 0.00053 & NFO & 34 \\
\hline 54747.72900 & 1 & 0.00020 & 0.00060 & NFO & 34 \\
\hline 54756.86680 & 2 & 0.00020 & 0.00006 & NFO & 34 \\
\hline 54758.69570 & 1 & 0.00020 & 0.00130 & NFO & 34 \\
\hline 54767.83230 & 2 & 0.00020 & 0.00044 & NFO & 34 \\
\hline 54778.79870 & 2 & 0.00020 & 0.00005 & NFO & 34 \\
\hline 54778.80030 & 2 & 0.00050 & 0.00155 & URSA & 34 \\
\hline 54789.76460 & 2 & 0.00030 & 0.00016 & NFO & 34 \\
\hline 54800.72950 & 2 & 0.00030 & 0.00127 & NFO & 34 \\
\hline 54811.69620 & 2 & 0.00030 & 0.00058 & NFO & 34 \\
\hline 54822.66220 & 2 & 0.00050 & 0.00058 & URSA & 34 \\
\hline
\end{tabular}

Notes.

Method: pg = photographic; vis = visual; ccd = ccd camera; URSA = URSA WebScope (ccd, $V$ filter); Sle $=$ Sleuth telescope (ccd, $r$ filter); NFO = NFO WebScope (ccd, $V$ filter).

References. (1) Strohmeier \& Bauernfeind 1968; (2) Reim 1957; (3) Grigorewski 1956; (4) Haeussler 1987; (5) Kreiner \& Winiarski 1977; (6) Mikulasek 1980; (7) Mikulasek 1985; (8) Locher 1982; (9) Paschke 1988; (10) Mikulasek \& Silhan 1988; (11) Mikulasek \& Silhan 1990; (12) Peter 1987; (13) Mikulasek et al. 1992; (14) Peter 1990; (15) Peter 1991; (16) Mikulasek 1994; (17) Peter 1992; (18) Molik 2007; (19) Paschke 1996; (20) Mikulasek 1996; (21) Blaettler 1997; (22) A. Paschke 2007, private communication; (23) Lacy 2002; (24) Sarounova \& Wolf 2005; (25) Dvorak 2003; (26) Lacy 2003b; (27) this paper; (28) Kotkova \& Wolf 2006; (29) Lacy 2004; (30) Kim et al. 2006; (31) Lacy 2006; (32) Lacy 2007; (33) Hübscher \& Walter 2007; (34) Lacy 2009.

\subsection{Spectroscopic Observations and Orbital Solution}

CO And was observed spectroscopically at the HarvardSmithsonian Center for Astrophysics (CfA) from 2004 January until 2008 December, with an echelle spectrograph and a photon-counting Reticon detector on the $1.5 \mathrm{~m}$ Tillinghast reflector at the F. L. Whipple Observatory, on Mount Hopkins, Arizona. A total of 30 spectra were collected in a single echelle order $45 \AA$ wide, centered at $5188.5 \AA$. The main features in this window are the lines of the $\mathrm{Mg}_{\mathrm{I}} \mathrm{b}$ triplet. The resolving power of this setup is $\lambda / \Delta \lambda \sim 35,000$, and the signal-to-noise ratios of the spectra range from 17 to 32 per resolution element of $8.5 \mathrm{~km} \mathrm{~s}^{-1}$.

Radial velocities were obtained with the two-dimensional cross-correlation algorithm TODCOR (Zucker \& Mazeh 1994), which is designed to minimize line-blending effects. The template spectra, one for each component of the binary, were chosen from an extensive library of synthetic spectra based on model atmospheres by R. L. Kurucz (see Latham et al. 2002). The parameters of these templates are the effective temperature $\left(T_{\text {eff }}\right)$, surface gravity $(\log g)$, rotational velocity $(v \sin i$ when seen in projection), and metallicity $([\mathrm{m} / \mathrm{H}])$. Following Torres et al. (2002), the optimum templates were selected by running extensive grids of cross-correlations with TODCOR exploring all possible parameter combinations, and seeking to maximize the average correlation value weighted by the strength of each exposure. Due to strong degeneracies among some of the template parameters, we initially set the surface gravity to $\log g=4.0$ for both stars, and the metallicity to solar. The best-fitting templates have $T_{\text {eff }}=6000 \mathrm{~K}$ and $v \sin i=25 \mathrm{~km} \mathrm{~s}^{-1}$ for both components, and these were adopted for the velocity determinations. In Section 5, we describe further experiments to quantify the correlations mentioned above, and to fine-tune the temperature, $v \sin i$, and metallicity determinations using other information available for the system. These parameter refinements have no effect on the velocities presented here. Our spectroscopic determinations indicate the components are very similar in all their properties. The temperature difference is formally only $70 \pm$ $50 \mathrm{~K}$ (the more massive star being marginally cooler), which is considerably smaller than the $250 \mathrm{~K}$ step in our template grid.

In addition to the velocities, we used TODCOR to obtain the light ratio between the components at the mean wavelength of our observations, following Zucker \& Mazeh (1994). The result is $L_{A} / L_{B}=1.012 \pm 0.025$ for the ratio between the marginally cooler and more massive component (star A), and the hotter but less massive star (B). Because the stars are so similar in temperature, the ratio should be very nearly the same at other wavelengths. We have nevertheless applied a small correction to the visual band based on synthetic spectra integrated over our spectral window and over the standard Johnson $V$ bandpass, which resulted in $\left(L_{A} / L_{B}\right)_{V}=1.016 \pm 0.025$. The same value holds for the Sloan $r$ band.

As in previous work with similar spectroscopic material (see, e.g., Torres \& Lacy 2009), we have used numerical simulations to assess the systematic errors in our velocities that may result from residual line blending in our narrow spectral window, or from shifts of the spectral lines in and out of this window as a function of orbital phase. Briefly, we generated synthetic composite spectra matching our observations by combining copies of the templates for each star, shifted to the appropriate velocities for each of the exposures as predicted by a preliminary orbital solution, and scaled to the observed light ratio. These synthetic observations were then processed with TODCOR in exactly the same way as the real spectra, and the resulting 
Table 5

Radial Velocities of CO And in the Heliocentric Frame

\begin{tabular}{|c|c|c|c|c|c|}
\hline HJD-2,400,000 & $\mathrm{RV}_{\mathrm{A}}\left(\mathrm{km} \mathrm{s}^{-1}\right)$ & $\mathrm{RV}_{\mathrm{B}}\left(\mathrm{km} \mathrm{s}^{-1}\right)$ & $(O-C)_{\mathrm{A}}\left(\mathrm{km} \mathrm{s}^{-1}\right)$ & $(O-C)_{\mathrm{B}}\left(\mathrm{km} \mathrm{s}^{-1}\right)$ & Phase \\
\hline 53014.6383 & -63.98 & 69.94 & 1.14 & 1.11 & 0.8740 \\
\hline 53192.9600 & -76.93 & 79.66 & 1.18 & 1.15 & 0.6580 \\
\hline 53216.9240 & 90.82 & -90.99 & 1.19 & 1.16 & 0.2139 \\
\hline 53218.9106 & -92.66 & 94.97 & 1.27 & 1.23 & 0.7574 \\
\hline 53271.8907 & 95.68 & -95.13 & 0.98 & 0.96 & 0.2513 \\
\hline 53273.8639 & -89.01 & 96.09 & 1.05 & 1.02 & 0.7911 \\
\hline 53278.8487 & 81.97 & -77.15 & 1.08 & 1.05 & 0.1548 \\
\hline 53282.8293 & 93.97 & -93.71 & 0.97 & 0.94 & 0.2438 \\
\hline 53302.7013 & -84.36 & 87.18 & 1.18 & 1.15 & 0.6802 \\
\hline 53308.7154 & 85.25 & -83.05 & 1.21 & 1.18 & 0.3255 \\
\hline 53335.7696 & -89.70 & 95.21 & 1.25 & 1.22 & 0.7268 \\
\hline 53370.6105 & 97.32 & -92.64 & 1.44 & 1.40 & 0.2583 \\
\hline 53576.9852 & -88.70 & 96.27 & 1.28 & 1.24 & 0.7168 \\
\hline 53627.9296 & -75.34 & 79.76 & 1.21 & 1.18 & 0.6538 \\
\hline 53631.8237 & -89.74 & 94.03 & 1.12 & 1.09 & 0.7191 \\
\hline 53688.7390 & 90.24 & -88.98 & 1.35 & 1.31 & 0.2896 \\
\hline 53695.7241 & 90.01 & -86.75 & 1.35 & 1.31 & 0.2006 \\
\hline 53723.6081 & -81.71 & 84.78 & 1.40 & 1.36 & 0.8289 \\
\hline 53989.9589 & -85.78 & 92.77 & 1.35 & 1.31 & 0.6952 \\
\hline 54044.7068 & -79.92 & 85.79 & 1.40 & 1.36 & 0.6727 \\
\hline 54046.8378 & 95.58 & -92.44 & 1.35 & 1.31 & 0.2557 \\
\hline 54101.5809 & 94.81 & -93.67 & 1.10 & 1.07 & 0.2319 \\
\hline 54108.6195 & 80.32 & -79.01 & 1.39 & 1.35 & 0.1575 \\
\hline 54395.7966 & -92.33 & 93.99 & 1.85 & 1.81 & 0.7213 \\
\hline 54457.6753 & -73.88 & 79.64 & 1.43 & 1.39 & 0.6496 \\
\hline 54483.6124 & -91.04 & 95.32 & 1.36 & 1.32 & 0.7453 \\
\hline 54485.6593 & 88.31 & -88.52 & 1.32 & 1.29 & 0.3053 \\
\hline 54742.8690 & -80.63 & 86.06 & 1.35 & 1.31 & 0.6709 \\
\hline 54777.8005 & 91.91 & -92.58 & 1.41 & 1.37 & 0.2272 \\
\hline 54812.7287 & -89.34 & 96.41 & 1.30 & 1.27 & 0.7826 \\
\hline
\end{tabular}

Note. All corrections mentioned in the text are included.

velocities were compared with the input shifts. The differences for CO And are typically smaller than $1 \mathrm{~km} \mathrm{~s}^{-1}$, and change systematically with phase as lines shift in and out of the $45 \AA$ spectral window. Because of this, they have a non-negligible effect on the absolute dimensions of the binary, so they were applied to the raw velocities as corrections. The effect on the absolute masses is $0.8 \%$, and the impact on the orbital semimajor axis used in computing the absolute radii is a smaller $0.25 \%$. Similar corrections for systematics were applied to the light ratio based on the same simulations, and are already included in the value reported earlier.

The stability of the zero point of the CfA velocity system was monitored by means of exposures of the dusk and dawn sky, and small run-to-run corrections were applied in the manner described by Latham (1992). The final velocities, including these offsets as well as the corrections for systematics described previously, are listed in Table 5 along with their uncertainties. The median value for the velocity errors is $1.3 \mathrm{~km} \mathrm{~s}^{-1}$ for both stars.

With these velocity measurements we carried out a weighted spectroscopic orbital solution for $\mathrm{CO}$ And, which is presented in Table 6 and shown graphically in Figure 5 along with the residuals. The ephemeris was held fixed at the results from Section 2, and the orbit was assumed to be circular since tests indicated that the eccentricity is not significant.

\section{LIGHT CURVE MODELING}

In order to remove the small nightly offsets in the differential photometry, we performed preliminary fits of the URSA and

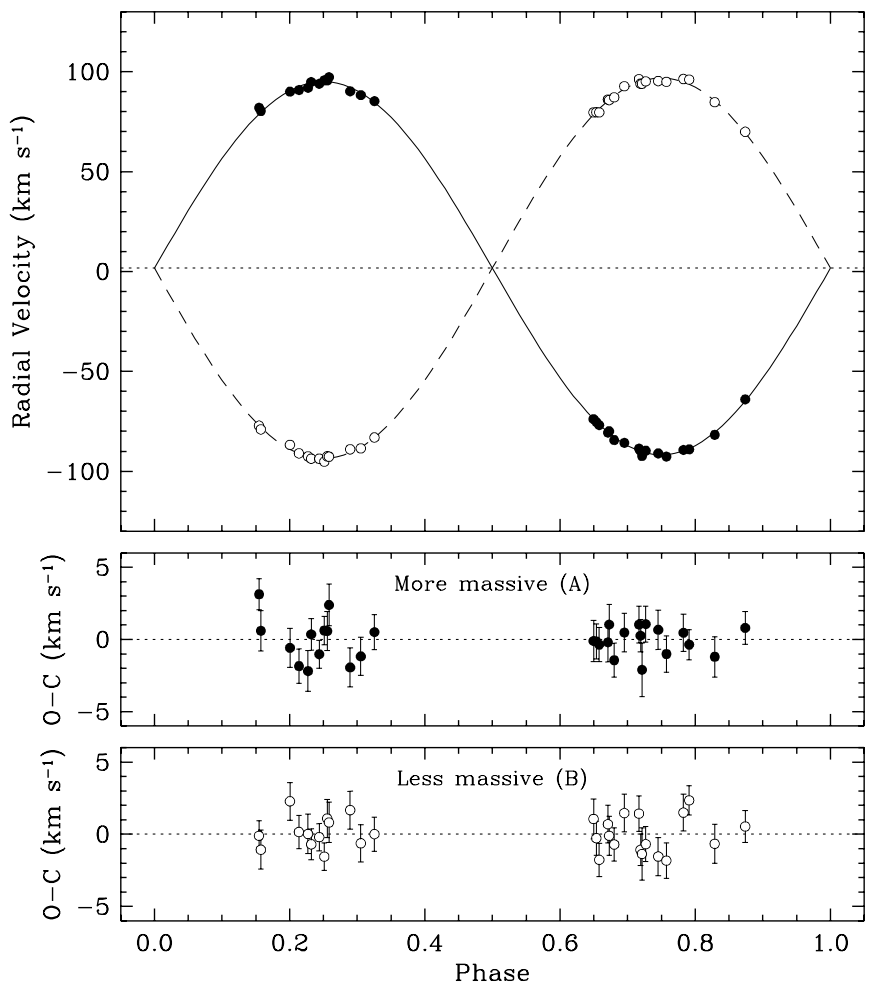

Figure 5. Measured radial velocities and computed orbit for CO And. Filled symbols correspond to the more massive and marginally cooler star (star A), and the dotted line represents the center-of-mass velocity. Error bars are smaller than the symbol size. $O-C$ residuals for both stars are shown at the bottom on an expanded scale. 
Table 6

Spectroscopic Solution for CO And

\begin{tabular}{|c|c|}
\hline Parameter & Value \\
\hline \multicolumn{2}{|c|}{ Adjusted quantities } \\
\hline$P$ (days) & 3.65533601 (fixed) \\
\hline$T_{I}(\mathrm{HJD})^{\mathrm{a}}$ & $2,453,764.44301$ (fixed) \\
\hline$K_{A}\left(\mathrm{~km} \mathrm{~s}^{-1}\right)$ & $93.40 \pm 0.24$ \\
\hline$K_{B}\left(\mathrm{~km} \mathrm{~s}^{-1}\right)$ & $95.24 \pm 0.24$ \\
\hline$\gamma\left(\mathrm{km} \mathrm{s}^{-1}\right)$ & $+1.66 \pm 0.16$ \\
\hline E & 0 (fixed) \\
\hline \multicolumn{2}{|c|}{ Derived quantities } \\
\hline$M_{A} \sin ^{3} i$ (solar masses) & $1.2837 \pm 0.0072$ \\
\hline$M_{B} \sin ^{3} i$ (solar masses) & $1.2589 \pm 0.0072$ \\
\hline$Q=M_{A} / M_{B}$ & $1.0197 \pm 0.0035$ \\
\hline$a_{A} \sin i\left(10^{6} \mathrm{~km}\right)$ & $4.695 \pm 0.012$ \\
\hline$a_{B} \sin i\left(10^{6} \mathrm{~km}\right)$ & $4.787 \pm 0.012$ \\
\hline$a \sin i($ solar radii $)$ & $13.630 \pm 0.025$ \\
\hline \multicolumn{2}{|c|}{ Other quantities pertaining to the fit } \\
\hline$N_{A} / N_{B}$ & 30 \\
\hline Time span (days) & 1798.1 \\
\hline$\sigma_{\mathrm{A}}\left(\mathrm{km} \mathrm{s}^{-1}\right)$ & 1.25 \\
\hline$\sigma_{\mathrm{B}}\left(\mathrm{km} \mathrm{s}^{-1}\right)$ & 1.22 \\
\hline
\end{tabular}

Note. ${ }^{a}$ This epoch corresponds to the eclipse of the less massive and marginally hotter star by the more massive one, which is the deeper minimum.

NFO light curves by using the Nelson-Davis-Etzel model (Popper \& Etzel 1981; Etzel 1981) as implemented in the EBOP code. The nightly residuals, which were typically less than $0.01 \mathrm{mag}$ and were uncorrelated with phase, were removed from each light curve. A few anomalous NFO values were also removed at this stage. These corrections improved the residual standard deviations of the URSA data by about $19 \%$, and those from NFO by a more significant $45 \%$.

The corrected differential photometry for $\mathrm{CO}$ And from the NFO, URSA, and Sleuth telescopes was analyzed with the same Nelson-Davis-Etzel light-curve model used above, as implemented in the JKTEBOP code of Southworth et al. (2007). This model is adequate for well-detached systems such as this with small relative radii (which are approximately 0.12 for both stars in CO And). The adjustable parameters are the cooler/hotter central surface brightness ratio $\left(J_{c}\right)$, the orbital inclination angle $(i)$, the sum of the relative radii in units of the semimajor axis $\left(r_{h}+r_{c}\right)$, the radius ratio $\left(k=r_{c} / r_{h}\right)$, a phase shift, and the magnitude at quadrature. Experiments solving also for the eccentricity $(e)$ and longitude of periastron $(\omega)$ did not yield statistically significant values of $e$, consistent with indications from spectroscopy, so this quantity was set to zero for the remainder of the analysis. Limb darkening was accounted for by adopting a linear law, consistent with our experience that more complicated laws do not improve the accuracy of the fits significantly with the amount and precision of the data at hand.

We have again checked on this assumption by doing numerical experiments fitting the NFO light curve data with a variety of nonlinear limb-darkening laws, including quadratic (Claret 2000; Claret \& Hauschildt 2003; Diaz-Cordoves et al. 1995), logarithmic (Claret 2000; Claret \& Hauschildt 2003; van Hamme 1993), and square root (Claret 2000; Claret \& Hauschildt 2003; Diaz-Cordoves et al. 1995; van Hamme 1993), using the observed values of temperature and $\log g$ to determine the coefficients. Our results are that the fitted values of radii are $0.2 \%$ smaller when using the nonlinear coefficients, and the inclination is smaller by 0.05 degrees. The adopted errors in the radii in Table 9 are more than five times larger than these errors, so we choose to use the simpler linear limb-darkening law with these data.

The linear limb-darkening coefficient $x$ was constrained to be the same for both stars, given their very similar properties. The gravity brightening coefficient $y$ was set to 0.34 for both stars as determined from their temperature and surface gravity, and the calculations by Claret (1998). The mass ratio was held fixed at the spectroscopic value of $q=M_{A} / M_{B}=1.0197$. Third light $\left(L_{3}\right)$ was also considered, and was found to be statistically significant for all three telescopes. We discuss this further below.

Initial solutions indicated a difficulty common in partially eclipsing systems with similar components, which is that while the sum of the radii is well constrained by the photometry, the radius ratio $k$ is not. Solutions with rather different values of $k$ deliver equally satisfactory fits to the data. As shown, e.g., by Popper (1984, 1987), Andersen et al. (1980, 1991), and others, an accurate external constraint such as that provided by the spectroscopic light ratio can serve to break the degeneracy. We have used this technique here.

Grids of JKTEBOP solutions were run for all three data sets over a range of fixed values of $k$ between 0.98 and 1.06. For each $k$ we determined the photometric light ratio $\left(L_{c} / L_{h}\right)$ along with all other elements, and then used the resulting relation between $k$ and $L_{c} / L_{h}$ to apply the spectroscopic constraint, $L_{A} / L_{B}=$ $\left(L_{c} / L_{h}\right)_{V}=\left(L_{c} / L_{h}\right)_{r}=1.016 \pm 0.025$, and determine the radius ratio. Other elements were interpolated to the same value of $k$. The procedure is illustrated for NFO in Figure 6, with the URSA and Sleuth relations being similar. We propagated the uncertainty in $L_{c} / L_{h}$ to $k$ and the rest of the photometric elements, as shown by the shaded areas in the figure. The results are summarized in Table 7 for the three telescopes.

We considered three sources of uncertainty in the light-curve elements, which are also listed in the table. One $\left(\varepsilon_{1}\right)$ is the error that comes directly from the spectroscopic constraint, propagated through to all interpolated variables. Another is a statistical component $\left(\varepsilon_{2}\right)$ due to the observational (photometric) uncertainties. We calculated it for each parameter using the option in JKTEBOP to perform Monte Carlo simulations, which provides a more realistic assessment than the formal errors otherwise returned by the program. We carried out 500 of these simulations, each time generating independent synthetic data sets assuming Gaussian error distributions. For the third contribution to the errors $\left(\varepsilon_{3}\right)$ we considered the interagreement between the solutions for the three telescopes (or only NFO and URSA for the wavelength-dependent parameters), and adopted half of the total range as a conservative uncertainty.

The adopted values of the light-curve elements are the weighted average of the three determinations in Table 7 (except for the passband-specific quantities $J_{c}, L_{3}$, and $x_{h}=x_{c}$ ), where the weights were computed as usual from the inverse square of the uncertainties, given by $\varepsilon=\operatorname{sqrt}\left(\varepsilon_{1}^{2}+\varepsilon_{2}^{2}\right)$. The final uncertainties were obtained by combining in quadrature the error of the weighted average with $\varepsilon_{3}$. These final results and uncertainties are given in Table 8.

As indicated earlier, the third light parameter from our fits is significant for all three telescopes, ranging from $1.4 \%$ (URSA) to $7.7 \%$ (Sleuth). At face value these suggest contamination of the light curve by another star. However, because of the large 
Table 7

Light-curve Solutions for CO And, Following the Application of the Spectroscopic Constraint on the Light Ratio

\begin{tabular}{|c|c|c|c|c|c|c|c|c|c|c|}
\hline \multirow[t]{2}{*}{ Element } & \multicolumn{3}{|c|}{$\mathrm{NFO}(\mathrm{V})$} & \multicolumn{3}{|c|}{ URSA (V) } & \multicolumn{3}{|c|}{ Sleuth (Sloan $r$ ) } & \multirow[t]{2}{*}{$\varepsilon_{3}$} \\
\hline & Param & $\varepsilon_{1}$ & $\varepsilon_{2}$ & Param & $\varepsilon_{1}$ & $\varepsilon_{2}$ & Param & $\varepsilon_{1}$ & $\varepsilon_{2}$ & \\
\hline$k$ & 1.025 & 0.013 & & 1.014 & 0.013 & & 1.019 & 0.013 & & \\
\hline$r_{h}+r_{c}$ & 0.24963 & 0.00055 & 0.00056 & 0.24810 & 0.00047 & 0.00090 & 0.25306 & 0.00050 & 0.00065 & 0.0027 \\
\hline$r_{h}$ & 0.12325 & 0.00053 & 0.00028 & 0.12319 & 0.00101 & 0.00045 & 0.12533 & 0.00055 & 0.00032 & 0.0011 \\
\hline$r_{c}$ & 0.12638 & 0.00107 & 0.00028 & 0.12491 & 0.00053 & 0.00045 & 0.12773 & 0.00106 & 0.00033 & 0.0014 \\
\hline$i$ & 87.001 & 0.035 & 0.036 & 86.953 & 0.021 & 0.059 & 86.808 & 0.033 & 0.055 & 0.096 \\
\hline$J_{c}$ & 0.96629 & 0.00090 & 0.00050 & 0.98815 & 0.00027 & 0.00082 & 0.97823 & 0.00075 & 0.00081 & $0.011(V)$ \\
\hline$L_{3}{ }^{\mathrm{a}}$ & 0.0369 & 0.0076 & 0.0071 & 0.0140 & 0.0061 & 0.0107 & 0.0769 & 0.0071 & 0.0091 & $0.011(V)$ \\
\hline$x_{h}=x_{c}$ & 0.649 & 0.035 & 0.034 & 0.475 & 0.032 & 0.059 & 0.711 & 0.032 & 0.039 & $0.087(V)$ \\
\hline$N$ & 5012 & & & 5941 & & & 9289 & & & \\
\hline Time span (days) & 1308.7 & & & 2639.0 & & & 55.9 & & & \\
\hline$\sigma(\mathrm{mmag})$ & 5.265 & 0.024 & & 9.4638 & 0.0038 & & 5.1508 & 0.0072 & & \\
\hline
\end{tabular}

Note. ${ }^{\text {a }} L_{c} / L_{h}=1.016 \pm 0.025$ and $L_{c}+L_{h}+L_{3}=1$ for each solution.

Table 8

Adopted Photometric Elements and Uncertainties for CO And

\begin{tabular}{lc}
\hline \hline Element & Value and Error \\
\hline$k$ & $1.0193,0.0093$ \\
$r_{h}+r_{c}$ & $0.2505,0.0025$ \\
$r_{h}$ & $0.1241,0.0012$ \\
$r_{c}$ & $0.1265,0.0015$ \\
$i(\operatorname{deg})$ & $86.94,0.10$ \\
$J_{c}(\mathrm{~V})$ & $0.979,0.011$ \\
$J_{c}(r)$ & $0.9782,0.0011^{\mathrm{a}}$ \\
$L_{3}(V)$ & $0.023,0.016^{\mathrm{b}}$ \\
$L_{3}(r)$ & $0.062,0.020^{\mathrm{b}}$ \\
$x_{h}=x_{c}(V)$ & $0.588,0.095$ \\
$x_{h}=x_{c}(r)$ & $0.711,0.050^{\mathrm{a}}$ \\
\hline
\end{tabular}

Notes.

a Internal errors only.

b Corrected for contamination from field stars (see the text). In both bands ( $V$ and $r$ ), $L_{c} / L_{h}=1.016 \pm 0.025$.

$L_{3}$ is defined in this model such that $L_{h}+L_{c}+L_{3}=1$.

photometric apertures we used, and the object's relatively low galactic latitude of $-15.8 \mathrm{deg}$, the chance of contamination from background sources is not negligible and must be considered before interpreting the $L_{3}$ values. The Sleuth aperture is the largest (30" radius), and within it there are no fewer than 10 faint stars listed in the USNO-B1.0 catalog (Monet et al. 2003). Of these, the seven closest are within the URSA aperture, and only the four closest affect NFO. Adding up the light contribution of these stars we determined corrections to the measured $L_{3}$ values, which are very small for NFO (0.0024) and URSA (0.0078), and more significant for Sleuth (0.0154). Errors in these values were estimated through Monte Carlo simulations, assuming a 0.4 mag uncertainty for the photographic magnitudes of these faint sources. The corrected values of $L_{3}$ for the $V$ and $r$ passbands are listed in Table 8 .

The stars in $\mathrm{CO}$ And are essentially spherical, and both eclipses are partial, with $76.9 \%$ of the light of Star B being lost at the primary eclipse, and $74.9 \%$ of the light of star A blocked at the secondary eclipse.

The limb-darkening coefficients $(x)$ determined in our solutions are not far from the theoretical values based on model atmospheres. Our estimate for the $V$ band, $0.588 \pm 0.095$, is intermediate between the values of 0.697 and 0.556 found in the tabulations by Claret (2000) and van Hamme (1993), respectively. Our determination for the Sloan $r$ band, $0.711 \pm 0.050$,
Table 9

Absolute Dimension of CO And

\begin{tabular}{lcc}
\hline \hline \multicolumn{1}{c}{ Parameter } & Cooler Star $(\mathrm{A})$ & Hotter Star $(\mathrm{B})$ \\
\hline Mass (solar masses) & $1.2892,0.0073$ & $1.2643,0.0073$ \\
Radius (solar radii) & $1.727,0.021$ & $1.694,0.017$ \\
Log $g(\mathrm{cgs})$ & $4.074,0.011$ & $4.0822,0.0089$ \\
$v \sin i\left(\mathrm{~km} \mathrm{~s}^{-1}\right)$ & $24.1,1.5$ & $23.8,1.5$ \\
$v \sin i(\mathrm{sync})\left(\mathrm{km} \mathrm{s}^{-1}\right)$ & $23.9,0.3$ & $23.4,0.2$ \\
$T_{\text {eff }}(\mathrm{K})$ & 6140,130 & 6170,130 \\
Log $L / L_{\text {sun }}$ & $0.580,0.038$ & $0.572,0.038$ \\
BC $(\mathrm{V})(\mathrm{mag})^{\mathrm{a}}$ & $-0.03,0.10$ & $-0.02,0.10$ \\
$M_{\text {bol }}(\mathrm{mag})$ & $3.281,0.096$ & $3.303,0.094$ \\
$M_{V}(\mathrm{mag})$ & $3.31,0.15$ & \\
$E(B-V)(\mathrm{mag})$ & $0.09,0.03$ & \\
Distance $(\mathrm{pc})$ & 377,25 & \\
$a(\mathrm{solar}$ radii) & $13.650,0.025$ & \\
{$[\mathrm{~m} / \mathrm{H}]$} & $+0.01,0.15$ & \\
\hline
\end{tabular}

Notes.

a Bolometric corrections from Flower (1996). The error includes contributions from the temperature uncertainties as well as an additional 0.10 mag we have added in quadrature, to be conservative. We have adopted $T_{\text {eff }}(\operatorname{Sun})=5780 \mathrm{~K}$ and $M_{\text {bol }}($ Sun $)=4.732$, to be consistent with the zero point of the bolometric corrections

is slightly larger than the value 0.639 given by Claret (2000), but perhaps still consistent given that our error estimate for this passband is strictly internal. For more extensive comparisons between theory and observation, the reader is referred to the work by Claret (2008).

\section{ABSOLUTE DIMENSIONS}

The combination of the spectroscopic and light-curve elements yields the absolute properties of $\mathrm{CO}$ And, which are summarized in Table 9. The relative errors in the masses are about $0.6 \%$, and in the radii $1.2 \%$ or better, which are among the best obtained for eclipsing binaries. Based on the discussion in Sections 2.3 and 3, we believe these errors to be realistic, and to therefore represent the true accuracy of the measurements (not merely the formal precision).

Effective temperatures are more difficult to determine accurately. While the light curves typically constrain the temperature ratio very well, the absolute scale is considerably more uncertain. The strong correlation between temperature and metallicity in our narrow spectral window prevents us from determining $T_{\text {eff }}$ (and $[\mathrm{m} / \mathrm{H}]$ ) directly from our spectra. However, with an 



Figure 6. Grid of photometric solutions with JKTEBOP for the NFO data of CO And, for a range of fixed values of $k=r_{c} / r_{h}$. Each panel shows the variation of one of the light-curve elements as a function of $k$, and the top left panel illustrates the application of the spectroscopic constraint on the light ratio $\left(L_{A} / L_{B}=L_{c} / L_{h}=\right.$ $1.016 \pm 0.025)$ to derive the final value of $k$. Values and corresponding uncertainties for all other elements interpolated to this value of $k$ are shown by the dashed line and shaded area, and are listed in Table 7. Similar grids were run for the URSA and Sleuth data.

independent estimate of the temperature such as from photometry, it is possible to resolve the degeneracy and determine both quantities. We follow this procedure here.

To better quantify the spectroscopic $T_{\text {eff }} /[\mathrm{m} / \mathrm{H}]$ correlation, we reran the grids of cross-correlations in Section 2.3 for a range of metallicities between $[\mathrm{m} / \mathrm{H}]=-1.0$ and +0.5 , in steps of 0.5 dex. We did this for two values of the surface gravity bracketing the final values in Table $9(\log g=4.0$ and 4.5), and then interpolated to the exact gravity for each star. Because the photometric temperatures described below correspond to the combined light, we combined the individual spectroscopic temperatures by weighting by the fractional luminosity of each star. In this way, we constructed a table of mean spectroscopic temperature as a function of metallicity. With an estimate of the mean temperature described below, the metallicity may then be read off.

External information on temperature can be obtained from color indices based on standard photometry for the combined light of $\mathrm{CO}$ And, together with color/temperature calibrations. Brightness measurements for the binary are available in a variety of photometric systems and are collected in Table 10, along with color indices for which reliable calibrations exist. The Two Micron All Sky Survey (2MASS) and Carlsberg Meridian Catalog measurements were taken out of eclipse, and we assume that the Tycho-2 and two-armed spiral shock (TASS) values are relatively unaffected by the eclipses given the large number of measurements. We adopt the weighted average of the three independent measurements in the Johnson $V$ band. The eight indices listed in Table 10 are not independent, but are still useful to give a sense of the scatter in the temperature results. For the color/temperature conversion we use three different calibrations by Ramírez \& Meléndez (2005), Casagrande et al. (2006), and González-Hernández \& Bonifacio (2009), all of which include correction terms for metallicity.

The chemical composition needed for those corrections is unknown to begin with, although it represents only a secondorder effect in the photometric $T_{\text {eff }}$. Reddening is potentially a much more important effect. We have estimated the reddening for CO And in two different ways. One is based on the Strömgren indices of Hilditch \& Hill (1975) listed in Table 10, and a table of standard indices such as that of Perry \& Johnston (1982). The result is $E(B-V)=0.116$. Another estimate may be obtained from dust maps such as those of Schlegel et al. (1998), which give a total reddening along the line of sight to the binary of $E(B-V)_{\text {tot }}=0.119$. The maps of Burstein \& Heiles (1982) give a nearly identical estimate of 0.118 , which is probably coincidental. We adopt the average of the two, $E(B-V)_{\mathrm{tot}}=0.118$. The fraction of this latter value that applies 
Table 10

Standard Photometry for CO And (Combined Light)

\begin{tabular}{lcc}
\hline \hline Passband/Color Index & Value and Error & Reference \\
\hline$B_{T}$ & $11.345,0.070$ & 1 \\
$V_{T}$ & $10.829,0.068$ & 1 \\
$J$ & $9.474,0.023$ & 2 \\
$H$ & $9.302,0.018$ & 2 \\
$K_{s}$ & $9.185,0.019$ & 2 \\
$V$ & $10.705,0.090$ & 3 \\
$I_{C}$ & $9.972,0.088$ & 3 \\
$V$ & $10.740,0.026$ & $4^{\mathrm{a}}$ \\
$V$ & $10.65,0.03$ & $5^{\mathrm{b}}$ \\
$u-b$ & $1.493,0.02$ & $5^{\mathrm{b}}$ \\
$v-b$ & $0.555,0.02$ & $5^{\mathrm{b}}$ \\
$b-y$ & $0.391,0.02$ & $5^{\mathrm{b}}$ \\
$B_{T}-V_{T}$ & $0.516,0.098$ & \\
$V-J$ & $1.228,0.039$ & \\
$V-H$ & $1.400,0.036$ & \\
$V-K_{S}$ & $1.517,0.036$ & \\
$J-K_{s}$ & $0.289,0.030$ & \\
$V_{T}-K_{s}$ & $1.644,0.071$ & \\
$V-I_{C}$ & $0.730,0.093$ & \\
\hline
\end{tabular}

Notes.

a Average of three determinations taken out of eclipse.

b Single measurement, so error is assigned conservatively.

References. (1) Tycho-2 (Høg et al. 2000); (2) 2MASS (Cutri et al. 2003); (3) TASS (Droege et al. 2006); (4) Carlsberg Meridian Catalog (1999-2006); (5) Hilditch \& Hill 1975

to $\mathrm{CO}$ And depends on the distance to the system, which is also an unknown quantity initially. The distance is a function of the known radii, the unknown temperatures, the apparent $V$ magnitude, bolometric corrections (which we take from Flower 1996 as a function of temperature), and the extinction $A(V)=$ $3.1 \times E(B-V)$. Once an estimate of the distance is obtained, we then take the average of the dust-map and Strömgren reddening values.

Most of the quantities mentioned above depend on one another in complicated ways, but it is possible to iterate this procedure to solve for the reddening, the distance, the individual temperatures, and the metallicity. Reddening-corrected temperature estimates from the eight color indices and three different calibrations typically agree within $150 \mathrm{~K}$. As indicated earlier, the light-curve fits provide strong information on the temperature ratio (or difference), and we use the central surface brightness ratio $J_{c}$ in the visual band along with Popper (1980) Table 1 to estimate $\Delta T_{\text {eff }}$. The procedure converges quickly, and leads to a consistent set of parameters that are listed in Table 9. The uncertainty in the individual temperatures, metallicity, and distance are dominated by the error in the reddening. Separate estimates of the distance for each component are in excellent accord (to within $1 \mathrm{pc})$, another sign of internal consistency. The temperature difference between the components is very small $(30 \pm$ $20 \mathrm{~K}$ ), and agrees with the rough value from spectroscopy but has less uncertainty. The final values of temperature are $T_{A}=$ $6140 \pm 130 \mathrm{~K}$ and $T_{B}=6170 \pm 130 \mathrm{~K}$, and the reddening is $E_{B-V}=0.09 \pm 0.03$.

The small amount of third light in our photometric measurements suggests the presence of another star in the system. It is possible that this is the same object responsible for the long-term trends in the eclipse timing residuals we reported in Section 2. The velocity residuals from our spectroscopic orbit show no evidence of a trend, but this is not surprising given the short interval of our observations (less than $5 \mathrm{yr}$ ) compared to the timescale of the timing variations (at least $100 \mathrm{yr}$; see Figure 4). We also examined our spectra using an extension of TODCOR to three dimensions (Zucker et al. 1995), but we see no sign of a third star. This is also not unexpected given that $L_{3}$ implies a 4 mag difference relative to the binary, in the visual band.

\section{COMPARISON WITH STELLAR MODELS}

Our accurate determination of the absolute properties of $\mathrm{CO}$ And, including the estimated value of metallicity, which is not typically known for eclipsing binaries, allows for a stringent test of recent stellar evolution models. We begin by using the calculations from the Yonsei-Yale series (Yi et al. 2001), with an updated convective core overshooting prescription described by Demarque et al. (2004). The masses of the components are large enough that a small convective core is beginning to develop in their centers, and overshooting has a measurable effect.

Using the interpolating routines provided by the modelers, we computed evolutionary tracks for the exact masses we measured. These are shown in the top panel of Figure 7 in a diagram of $\log g$ versus $T_{\text {eff }}$. There is a small uncertainty in the location of these tracks that comes from the observational errors in the masses; we represent this by the shaded areas in the figure. The chemical composition of the models was adjusted to provide the best match with the observations, and indicates $Z=0.0148$, corresponding to $[\mathrm{Fe} / \mathrm{H}]=-0.09$. This is in good agreement with the measured metallicity of $[\mathrm{m} / \mathrm{H}]=+0.01 \pm 0.15$. The age determined from the models is $3.65 \mathrm{Gyr}$, based on this diagram. The corresponding isochrone is shown with the dashed line. We note that while the errors in the absolute temperatures are relatively large compared to the separation between the tracks $(130 \mathrm{~K})$, the temperature difference from photometry is much better determined, and is indicated in the figure $\left(\sigma\left[\Delta T_{\text {eff }}\right]\right)$. Both our photometric estimate and our spectroscopic estimate indicate the components are nearly indistinguishable in temperature, the more massive star being marginally cooler. The models also indicate a very small difference, although an accurate estimate is made difficult by limitations in the interpolating scheme that lead to a jagged appearance of the isochrones. This is seen in the lower panels of Figure 7, which nevertheless illustrate the good overall fit to the individual radii and effective temperatures as a function of the best-measured property, the mass.

The stars in CO And are seen to be very near the end of their main-sequence phase, just prior to leftward turn ("blue hook") that precedes the stage of their evolution across the Hertzsprung gap in which they burn hydrogen in a shell. It is precisely at their present phase that the consequences of overshooting are seen more clearly and are better tested, since it has the effect of extending the hydrogen-burning main sequence toward lower temperatures in this diagram. The implementation of overshooting in the Yonsei-Yale models, parameterized in terms of a simple multiplicative scale factor $\alpha_{\mathrm{ov}}$ to the local pressure scale height, is such that $\alpha_{\mathrm{ov}}$ ramps up gradually from zero (when there is no convective core at all) up to a maximum of $\alpha_{\mathrm{ov}}=0.2$ as the mass of the model increases. At the metallicity and mass of $\mathrm{CO}$ And, the $\alpha_{\mathrm{ov}}$ in the interpolated tracks is approximately $0.08-0.09$, or about half of the maximum value. The location of the binary in Figure 7 is consistent with this value. If $\alpha_{\text {ov }}$ were much smaller than this, the theoretical main sequence would retract toward hotter temperatures, leaving the stars in an unphysical position in the diagram. 

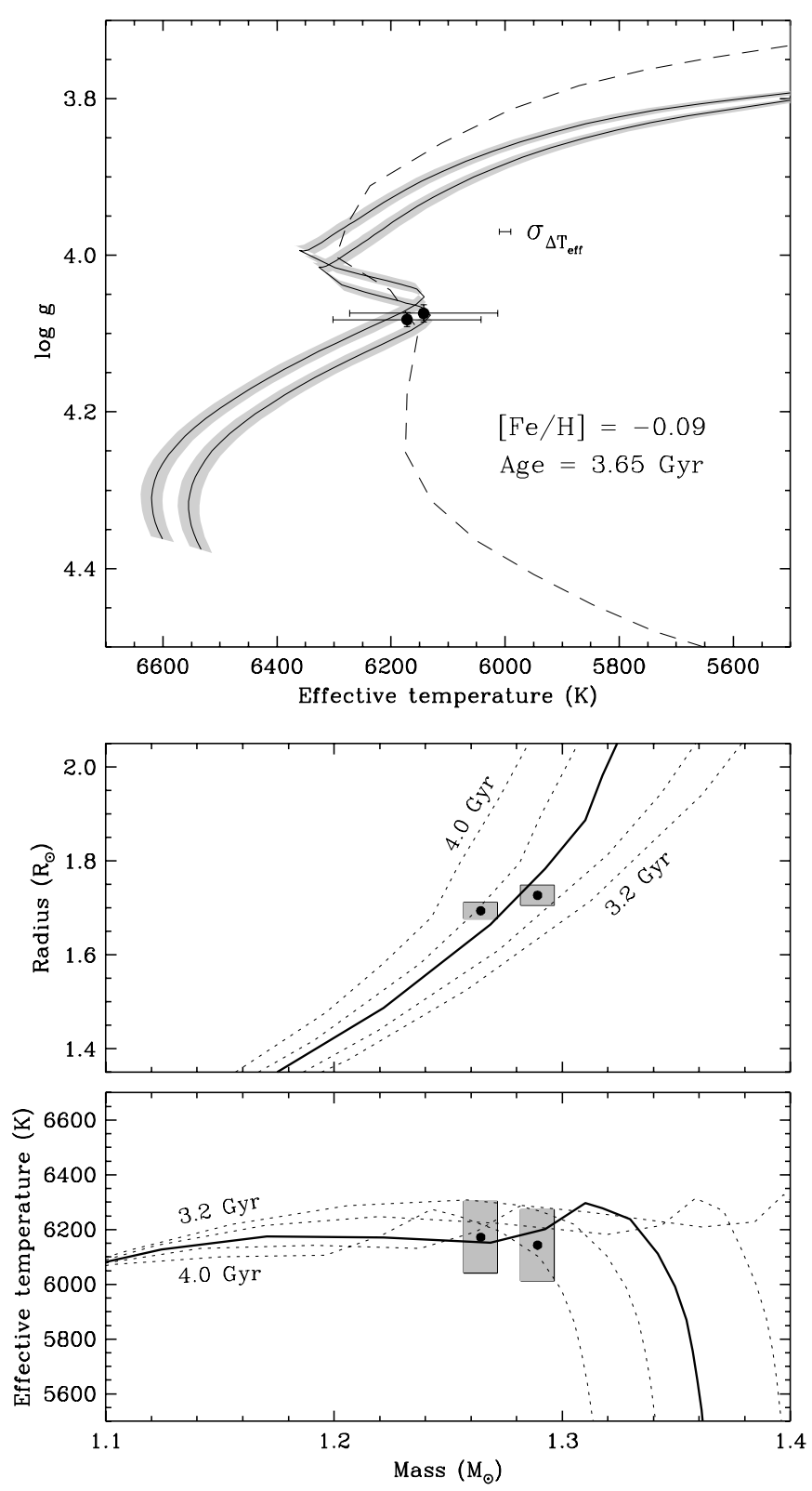

Figure 7. Top: observations of $\mathrm{CO}$ And compared against evolutionary tracks from the Yonsei-Yale series (Yi et al. 2001; Demarque et al. 2004), calculated for the exact masses we measure (solid lines). The shaded areas represent the uncertainty in the location of the tracks that comes from the mass uncertainties. The best-fitting age and metallicity are $3.60 \mathrm{Gyr}$ and $Z=0.0148$ (corresponding to $[\mathrm{Fe} / \mathrm{H}]=-0.09$ in these models). The corresponding isochrone is shown by the dashed line. Also indicated is the observational uncertainty $\left(\sigma_{\Delta T \text { eff }}\right)$ in the temperature difference between the components, which is much smaller than the errors in the absolute temperatures. Middle: radius as a function of mass, along with isochrones for the best-fit metallicity between $3.2 \mathrm{Gyr}$ and $4.0 \mathrm{Gyr}$ in steps of 0.2 Gyr. The thick line corresponds to the best-fit age, and error bars are indicated by the shaded regions. Bottom: the same as above, for effective temperature vs. mass.

We examined also the match between the CO And properties and the stellar evolution models of the Victoria-Regina group (VandenBerg et al. 2006), which use a different prescription for overshooting based on a parameterized version of the Roxburgh criterion (Roxburgh 1978, 1989). The comparison with the corresponding isochrones is illustrated in Figure 8. We find a very good concordance for a metallicity of $[\mathrm{Fe} / \mathrm{H}]=-0.11$, once again consistent with our spectroscopic estimate, and an age of $3.40 \mathrm{Gyr}$. The lower panels of the figure show the
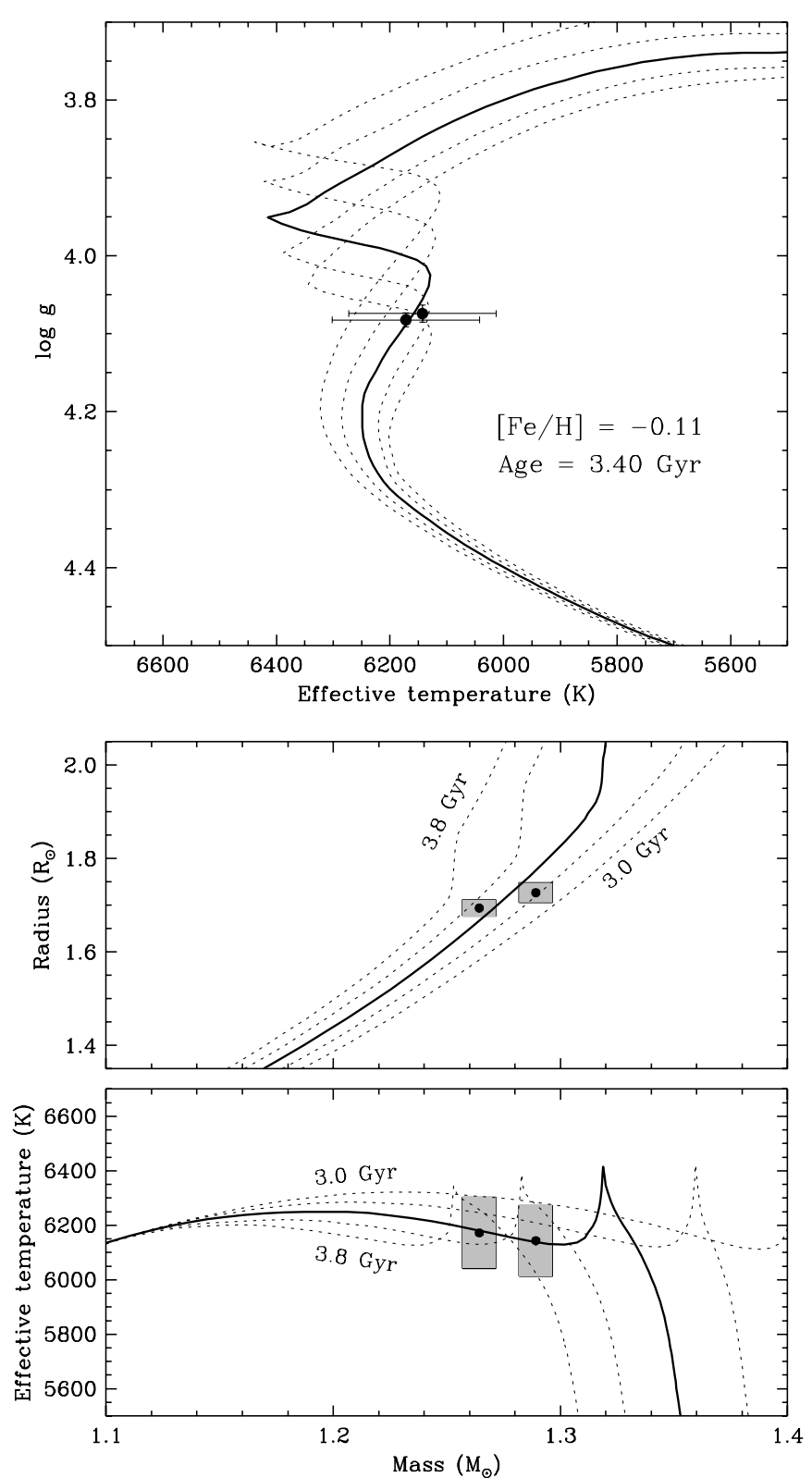

Figure 8. Observations of $\mathrm{CO}$ And compared against model isochrones from the Victoria-Regina series (VandenBerg et al. 2006). Top: isochrones for the best-fit metallicity of $[\mathrm{Fe} / \mathrm{H}]=-0.11$ and ages from $3.0 \mathrm{Gyr}$ (left) to $3.8 \mathrm{Gyr}$ (right), in steps of $0.2 \mathrm{Gyr}$. The best matching age of $3.40 \mathrm{Gyr}$ is indicated with the thick line. Middle: radius as a function of mass. Bottom: temperature as a function of mass.

comparison with the radii and temperatures. The models indicate a temperature difference of $41 \mathrm{~K}$ (the more massive star being cooler), in excellent agreement with our estimate of $30 \pm 20 \mathrm{~K}$.

In order to explore the impact of convective core overshooting more quantitatively, we have made use of the Granada models by Claret (2004), which use the same prescription as the Yonsei-Yale models and allow greater flexibility in adjusting the overshooting parameter. The metallicity that best matches the observations for these models is $Z=0.016$, corresponding to $[\mathrm{Fe} / \mathrm{H}]=-0.03$. Once again this is consistent with the spectroscopically measured composition. In Figure 9, we show the evolutionary tracks for a range of values of $\alpha_{\mathrm{ov}}$, including calculations with no overshooting at all (top panel). The observations are clearly inconsistent with $\alpha_{\mathrm{ov}}=0.00$, and from the lower panels it appears that the minimum value that is able to match 

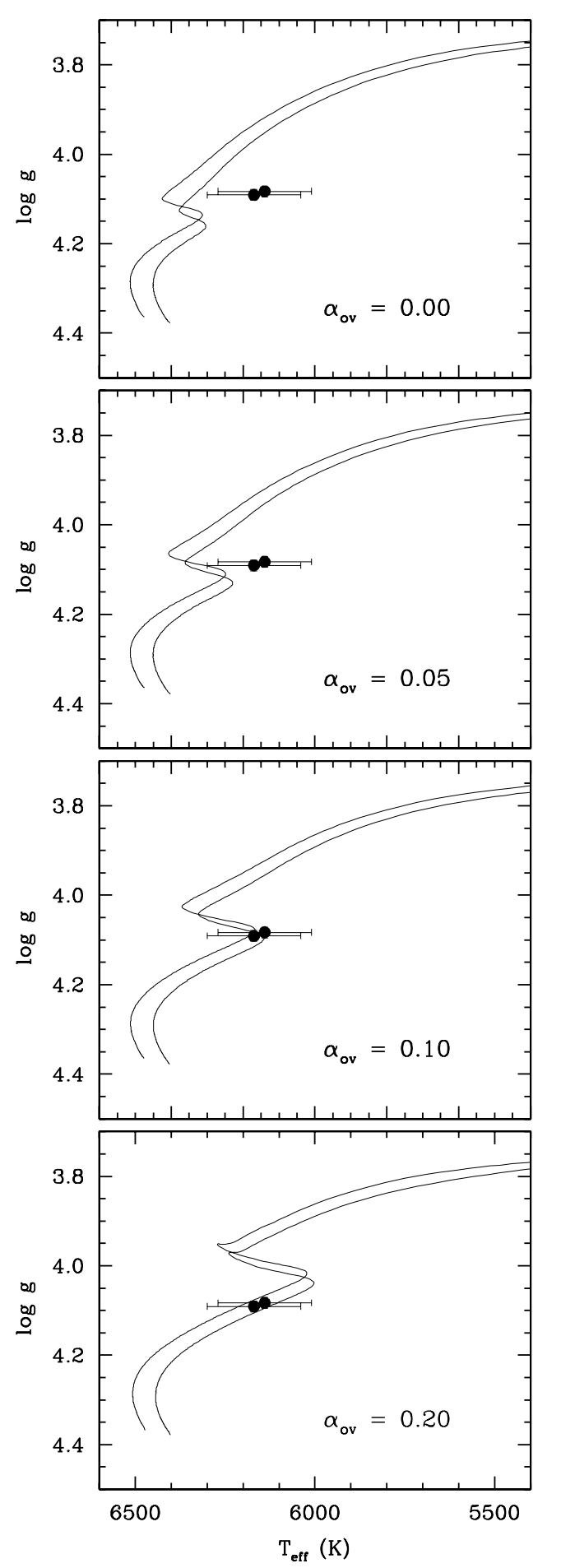

Figure 9. Comparison of the observations for $\mathrm{CO}$ And against stellar evolution models by Claret (2004), for the best-fit composition of $Z=0.016$ (corresponding to $[\mathrm{Fe} / \mathrm{H}]=-0.03$ ). The different panels show the effect of changing the overshooting parameter $\alpha_{\mathrm{ov}}$. The best-fit age from the models with $\alpha_{\mathrm{ov}}=0.10$ is $3.8 \mathrm{Gyr}$, and for $\alpha_{\mathrm{ov}}=0.20$ is $3.9 \mathrm{Gyr}$.

the observations is around $\alpha_{\mathrm{ov}}=0.10$, or slightly higher. This is not very different than the value used in the Yonsei-Yale models, although it is also clear by comparison with the top panel in Figure 7 that there are differences in the tracks from these two models even for similar amounts of overshooting. These differences have to do mostly with the details of the physics in each set of calculations. For example, the Yonsei-Yale models include helium diffusion, while the Granada models do not. There are also differences in the equation of state, in the radiative opacities, and in the numerical methods. In any case, we conclude that the observations point to a lower limit for $\alpha_{\text {ov }}$ of about 0.10 for the Granada models, and perhaps somewhat smaller for the Yonsei-Yale models, and that they clearly exclude zero overshooting. The age of the system implied by the Granada models for $\alpha_{\mathrm{ov}}=0.10$ is $3.8 \mathrm{Gyr}$, and for $\alpha_{\mathrm{ov}}=0.20$ it is $3.9 \mathrm{Gyr}$. These are both slightly older than derived from the other models.

Additional stellar properties provided by the stellar evolution models include the absolute magnitudes in various passbands. These may be compared with the measured brightness of $\mathrm{CO}$ And, and used as a check on the distance reported earlier. For this test, we selected the best-fit Yonsei-Yale isochrone discussed above. After converting the near-infrared 2MASS magnitudes in Table 10 to the ESO system of the isochrone with the transformations of Carpenter (2001), and correcting all measures for interstellar extinction, the average distance for the $V, I_{C}, J, H$, and $K$ passbands is $370 \mathrm{pc}$, very close to our previous estimate.

Finally, the stellar evolution models also allow us to make a rough estimate of the mass of the third star in $\mathrm{CO}$ And implied by the $L_{3}$ parameters in the light-curve fits. Using the same best-fit Yonsei-Yale isochrone from above, we find that the measured $V$-band and $r$-band third light parameters are consistent with being produced by a star of approximately 0.8 solar masses, or slightly larger. If this object is physically associated with the binary and is responsible for the light-travel time effect seen in Figure 4, the semimajor axis of its orbit around $\mathrm{CO}$ And would correspond to an angular separation of 0.09 arcsec if the period is $100 \mathrm{yr}$, or $0.25 \operatorname{arcsec}$ if $P=500 \mathrm{yr}$. Confirmation should be possible with high-resolution imaging techniques.

\section{CONCLUDING REMARKS}

As a result of our intensive spectroscopic and photometric monitoring, the absolute masses of $\mathrm{CO}$ And are now determined to $0.6 \%$ accuracy and the radii to $1.2 \%$, and these are among the best available for any eclipsing binary. In addition, we have established the effective temperatures, as well as estimating the overall metallicity. The latter quantity has not been determined for many eclipsing binaries, and is important because it reduces the degrees of freedom in the comparison with theory. Unfortunately, the precision of $[\mathrm{Fe} / \mathrm{H}]$ (and $T_{\text {eff }}$ to some extent) is limited in this case by poor knowledge of interstellar extinction in the direction of the star. This could perhaps be remedied with additional and more accurate absolute photometric observations. Nevertheless, the combination of $M, R$, $T_{\text {eff }}$, and $[\mathrm{Fe} / \mathrm{H}]$ provides unusually strong constraints on stellar evolution theory. Comparison with current models yields an excellent fit to the observations, and indicates the stars are near the end of their main-sequence phase, at an age of about 3.6 Gyr. Because of their position near the blue hook at the end of the main sequence, we are able to put constraints on the degree of core overshooting, which is clearly non-zero in this case.

With masses around 1.3 solar masses, $\mathrm{CO}$ And is an important addition to well-studied main-sequence systems in the mass interval (approximately1.1-1.7 solar masses) where convective overshooting is ramped up in evolutionary models. Only GX Gem and BW Aqr are listed by Torres \& Lacy (2009) to be in a similar evolutionary state, both of them more massive than CO And.

We thank P. Berlind, M. Calkins, G. Esquerdo, D. Latham, and R. Stefanik for their help in obtaining the spectroscopic 
observations of CO And used in this work. G.T. acknowledges partial support for this project from NSF grant AST-0708229. This research has made use of the SIMBAD database and the VizieR catalog access tool of NASA's Astrophysics Data System Abstract Service, both operated at CDS, Strasbourg, France and of data products from the 2MASS, which is a joint project of the University of Massachusetts and the Infrared Processing and Analysis Center/California Institute of Technology, funded by NASA and the NSF. C.H.S.L. thanks Dr. A. William Neely for operating and maintaining the NFO WebScope, and for preliminary processing of NFO images. This research was supported in part by NASA under grant NNG05GJ29G (issued through the Origins of Solar Systems Program).

\section{REFERENCES}

Andersen, J., Clausen, J. V., \& Nordström, B. 1980, in IAU Symp. 88, Close Binary Stars: Observations and Interpretation, ed. M. J. Plavec, D. M. Popper, \& R. K. Ulrich (Reidel: Dordrecht), 81

Andersen, J., Clausen, J. V., Nordström, B., Tomkin, J., \& Mayor, M. 1991, A\&A, 246, 99

Blaettler, E. 1997, BBSAG Bull., 114

Burstein, D., \& Heiles, C. 1982, AJ, 87, 1165

Carlsberg Meridian Catalog 1999-2006, Copenhagen University Obs., Institute of Astronomy, Cambridge, UK, Real Instituto y Observatorio de la Armada en San Fernando

Carpenter, J. M. 2001, AJ, 121, 2851

Casagrande, L., Portinari, L., \& Flynn, C. 2006, MNRAS, 373, 13

Claret, A. 1998, A\&AS, 131, 395

Claret, A. 2000, A\&A, 363, 1081

Claret, A. 2004, A\&A, 424, 919

Claret, A. 2008, A\&A, 482, 259

Claret, A., \& Hauschildt, P. H. 2003, A\&A, 412, 241

Cutri, R. M., et al. 2003, 2MASS All-Sky Catalog of Point Sources, Univ. of Massachusetts and Infrared Processing and Analysis Center (IPAC/ California Institute of Technology)

Demarque, P., Woo, J.-H., Kin, Y.-C., \& Yi, S. K. 2004, ApJS, 155, 667

Diaz-Cordoves, J., Claret, A., \& Gimenez, A. 1995, A\&AS, 110, 329

Droege, T. F., Richmond, M. W., Sallman, M. P., \& Creager, R. P. 2006, PASP, 118,1666

Dvorak, S. W. 2003, IBVS, 5378, 1

Etzel, P. B. 1981, in Photometric and Spectroscopic Binary Systems, ed. C. B. Corling \& E. Z. Kopal (Dordrecht: Reidel), 111

Flower, P. J. 1996, ApJ, 469, 355

Geyer, E., Kippenhahn, R., \& Strohmeier, W. 1955, Kleine Veroff. RemeisSternwarte Bamberg, 11,

González-Hernández, J. I., \& Bonifacio, P. 2009, A\&A, 497, 497

Grauer, A. D., Neely, A. W., \& Lacy, C. H. S. 2008, PASP, 120, 992

Grigorewski, V. M. 1956, Astron. Circ. Kasan, 170, 17

Haeussler, K. 1987, in Harthaer Beobachtungs-Zirkular (Hartha: Sternwarte Hartha), 81

Hilditch, R. W., \& Hill, G. 1975, MmRAS, 79, 101

Høg, E., et al. 2000, A\&A, 355, 27

Hübscher, J., \& Walter, F. 2007, IBVS, 5761

Kim, C., Lee, C., Yoon, Y., Park, S., Kim, D., Cha, S., \& Won, J. 2006, IBVS, 5694

Kotkova, L., \& Wolf, M. 2006, IBVS, 5676
Kreiner, J. M., \& Winiarski, M. 1977, IBVS, 1255

Kwee, K. K., \& van Woerden, H. 1956, Bull. Astron. Inst. Neth., 12, 327

Lacy, C. H. S. 2002, IBVS, 5357, 1

Lacy, C. H. S. 2003a, Astronomy Education Review (aer.noao.edu), 2, 1

Lacy, C. H. S. 2003b, IBVS, 5487, 1

Lacy, C. H. S. 2004, IBVS, 5577, 1

Lacy, C. H. S. 2006, IBVS, 5670, 1

Lacy, C. H. S. 2007, IBVS, 5764, 1

Lacy, C. H. S. 2009, IBVS, 5910, 1

Lacy, C. H. S., Hood, B., \& Straughn, A. 2001, IBVS, 5067

Latham, D. W. 1992, in IAU Colloq. 135, ASP Conf. Ser. 32, Complementary Approaches to Double and Multiple Star Research, ed. H. A. McAlister \& W. I. Hartkopf (San Francisco, CA: ASP), 110

Latham, D. W., Stefanik, R. P., Torres, G., Davis, R. J., Mazeh, T., Carney, B. W., Laird, J. B., \& Morse, J. A. 2002, AJ, 124, 1144

Locher, K. 1982, BBSAG Bull., 63

Mikulasek, Z. 1980, Contrib. N. Copernicus Obs. Planetarium Brno, 23

Mikulasek, Z. 1985, Contrib. N. Copernicus Obs. Planetarium Brno, 26

Mikulasek, Z. 1994, Contrib. N. Copernicus Obs. Planetarium Brno, 31

Mikulasek, Z. 1996, Contrib. N. Copernicus Obs. Planetarium Brno, 32

Mikulasek, Z., \& Silhan, J. 1988, Contrib. N. Copernicus Obs. Planetarium Brno, 28

Mikulasek, Z., \& Silhan, J. 1990, Contrib. N. Copernicus Obs. Planetarium Brno, 29

Mikulasek, Z., Silhan, J., \& Zejda, M. 1992, Contrib. N. Copernicus Obs. Planetarium Brno, 30

Molik, P. 2007, Open Eur. J. Var. Stars, 60

Monet, D. G., et al. 2003, AJ, 125, 984

O'Donovan, F. T., Charbonneau, D., \& Kotredes, L. 2004, in AIP Conf. Proc. 713, The Search for Other Worlds, ed. S. S. Holt \& D. Deming (Melville, NY: AIP), 1690

Paschke, A. 1988, BBSAG Bull., 86

Paschke, A. 1996, BBSAG Bull., 112

Perry, C. L., \& Johnston, L. 1982, ApJS, 50, 451

Peter, H. 1987, BBSAG Bull., 83

Peter, H. 1990, BBSAG Bull., 93

Peter, H. 1991, BBSAG Bull., 97

Peter, H. 1992, BBSAG Bull., 99

Popper, D. M. 1980, ARA\&A, 18, 115

Popper, D. M. 1984, AJ, 89, 1057

Popper, D. M. 1987, AJ, 93, 672

Popper, D. M., \& Etzel, P. B. 1981, AJ, 86, 102

Ramírez, I., \& Meléndez, J. 2005, ApJ, 626, 446

Reim, W. 1957, Kleine Veroff. Remeis-Sternwarte Bamberg, 17, 1

Roxburgh, I. W. 1978, A\&A, 65, 281

Roxburgh, I. W. 1989, A\&A, 211, 361

Sarounova, L., \& Wolf, M. 2005, IBVS, 5594

Schlegel, D. J., Finkbeiner, D. P., \& Davis, M. 1998, ApJ, 500, 525

Selman, F. J. 2004, Proc. SPIE, 5493, 453

Southworth, J., Bruntt, H., \& Buzasi, D. L. 2007, A\&A, 467, 1215

Strohmeier, W., \& Bauernfeind, H. 1968, Veroff. Remeis-Sternwarte Bamberg, 7,72

Torres, G., \& Lacy, C. H. S. 2009, AJ, 137, 507

Torres, G., Neuhäuser, R., \& Guenther, E. W. 2002, AJ, 123, 1701

VandenBerg, D. A., Bergbusch, P. A., \& Dowler, P. D. 2006, ApJS, 162, 375 van Hamme, W. 1993, AJ, 106, 2096

Yi, S., Demarque, P., Kin, Y.-C., Lee, Y.-W., Ree, C. H., Lejeune, T., \& Barnes, S. 2001, ApJS, 136, 417

Zucker, S., \& Mazeh, T. 1994, ApJ, 420, 806

Zucker, S., Torres, G., \& Mazeh, T. 1995, ApJ, 452, 863 